\title{
Discrete diffraction and Bloch oscillations in non-Hermitian frequency lattices induced by complex photonic gauge fields
}

\author{
Chengzhi Qin, ${ }^{1,2}$ Bing Wang $\odot,{ }^{1, *}$ Zi Jing Wong, ${ }^{2, \dagger}$ Stefano Longhi, ${ }^{3,4, \star}$ and Peixiang Lu ${ }^{1,5, \S}$ \\ ${ }^{1}$ School of Physics and Wuhan National Laboratory for Optoelectronics, \\ Huazhong University of Science and Technology, Wuhan 430074, China \\ ${ }^{2}$ Department of Aerospace Engineering, Texas A\&M University, College Station, Texas 77843, USA \\ ${ }^{3}$ Dipartimento di Fisica, Politecnico di Milano, Piazza Leonardo da Vinci 32, 20133 Milano, Italy \\ and Istituto di Fotonica e Nanotecnologie, Consiglio Nazionale delle Ricerche, Piazza Leonardo da Vinci 32, 20133 Milano, Italy \\ ${ }^{4}$ IFISC (UIB-CSIC), Instituto de Fisica Interdisciplinar y Sistemas Complejos - Palma de Mallorca, E-07122 Spain \\ ${ }^{5}$ Hubei Key Laboratory of Optical Information and Pattern Recognition, Wuhan Institute of Technology, Wuhan 430205, China
}

(Received 18 November 2019; revised manuscript received 24 January 2020; accepted 24 January 2020; published 12 February 2020)

\begin{abstract}
Non-Hermitian lattice systems with unconventional transport phenomena and topological effects have attracted intensive attention recently. Non-Hermiticity is generally introduced by engineering on-site gain/loss distribution or inducing asymmetric couplings by applying an imaginary gauge field. Here, we extend the concept of non-Hermitian lattices from spatial to frequency dimension and emulate various non-Hermitian transport phenomena arising from asymmetric coupling in synthetic dimension. The non-Hermitian frequency lattice is created by introducing complex gauge potentials through appropriate complex modulations in a slab waveguide. This complex gauge potential can induce asymmetric couplings among spectral modes and give rise to various non-Hermitian transport phenomena such as amplified and decayed frequency diffraction, refraction and non-Hermitian Bloch oscillations. The latter manifest themselves as both power oscillation and asymmetric oscillation patterns, and can be exploited to probe in the bulk the non-Hermitian skin effect. Frequency-domain Bloch oscillations with both exponentially growing oscillation amplitude and energy are also predicted. Our results pave the way towards emulating non-Hermitian transport phenomena and topological effects in synthetic dimension on a photonic platform, with potential applications to spectral manipulation of optical signals and energy harvesting.
\end{abstract}

DOI: 10.1103/PhysRevB.101.064303

\section{INTRODUCTION}

Photonic lattice systems, such as photonic crystals, metamaterials, and coupled waveguide arrays, provide ideal platforms to emulate wave transport dynamics of electrons in solid-state systems [1-5]. Typical transport phenomena include the force-free discrete diffraction and force-driven Bloch oscillations [6-11]. In recent years, the idea of nonHermiticity has emerged as a paradigm to mold the flow of light in photonic lattices. Examples include the control of light spreading from ballistic to diffusive in non-Hermitian waveguide arrays [12] and nonreciprocal Bloch oscillations in parity-time $(P T)$ symmetric lattice potentials $[13,14]$. From a practical perspective, the presence of non-Hermiticity also offers functionalities to control light energy dynamics, such as realizing selective light amplification and/or absorption as well as energy harvesting purposes [15-20].

Basically, there are two approaches to introduce nonHermiticity into photonic lattices: the first one is to apply

\footnotetext{
*wangbing@hust.edu.cn

†zijing@tamu.edu

${ }^{\ddagger}$ stefano.longhi@polimi.it

§lupeixiang@hust.edu.cn
}

on-site gain/loss distribution, as typically exploited in traditional $P T$-symmetric systems [21-23]. The second one is to introduce asymmetric couplings between adjacent lattice sites. The concept of asymmetric coupling dates back to the Hatano-Nelson model in the 1990s [24,25], where a nonHermitian delocalization transition in the Anderson model was predicted to occur by application of an imaginary gauge field inducing asymmetric left/right hopping on the lattice. More recently, non-Hermitian lattices with asymmetric couplings are attracting a rapidly growing attention for their unusual spectral and topological properties when subjected to open boundary conditions. A peculiar feature of such systems is to show so-called non-Hermitian skin effect (NHSE), i.e., the squeezing of bulk states to the edges, breakdown of bulk-boundary correspondence based on Bloch topological invariants, and non-Bloch symmetry breaking phase transitions [26-44]. Asymmetric coupling is also of special interest in laser design, for example for the realization of topological lasers [45] and robust phase locking in laser arrays [46]. Hence, the implementation of asymmetric hopping in photonics is becoming a quest of great relevance. Despite the facility in introducing non-Hermiticity in optics, the realization of asymmetric couplings, or equivalently imaginary gauge fields requires rather complex auxiliary coupling elements [47,48], which remains technically a quite challenging task. 
In this work, we extend the concept of non-Hermitian lattice to frequency dimension and achieve various nonHermitian transport phenomena, suggesting also a method to probe the NHSE by means of frequency Bloch oscillations. Synthetic dimension refers to an artificial lattice formed by coupling a set of modes with equally spaced parameters like frequency, time or momentum [49-58], making it possible to emulate higher-dimensional physics in lower-dimensional physical structures. Here we apply complex index modulation to introduce photonic transitions in a slab waveguide and create a frequency lattice. Then the complex gauge potential can be introduced by choosing appropriate amplitudes and phases for the real and imaginary modulation parts, through which we realize amplified and decayed frequency diffraction and refraction. By introducing an effective force through a wave number mismatch, we also achieve non-Hermitian Bloch oscillations [59] in the frequency lattice, showing both energy oscillation and asymmetric oscillation patterns. Remarkably, the non-Hermitian Bloch oscillation dynamics can reveal the existence or not the NHSE [26-44], i.e., they provide a bulk probing method to detect squeezing of bulk modes at the edges in lattices with open boundary conditions (OBCs). Moreover, we generalize the effective force itself to the complex regime and realize amplified Bloch oscillations with both exponentially growing oscillation amplitude and energy. Finally, a concrete modulator structure with judiciously designed modulation profiles is proposed to realize both the complex gauge potential and effective force.

\section{RESULTS AND DISCUSSIONS}

\section{A. Model and discrete diffraction in non-Hermitian frequency lattice}

In this section we describe our basic photonic system to implement a non-Hermitian frequency lattice, in which asymmetric hopping between adjacent sites is realized by a synthetic gauge field via combined phase and amplitude modulations. As shown in Fig. 1(a), we consider a $\mathrm{LiNbO}_{3}$ slab waveguide subject to a traveling-wave complex index modulation $n(z, t)=n_{0}+\Delta n_{1} \cos \left(\Omega t-q_{m} z+\phi_{1}\right)+$ $i \Delta n_{2} \cos \left(\Omega t-q_{m} z+\phi_{2}\right)$ [Fig. 1(b)], where $n_{0}$ is the background refractive index, $\Omega$ and $q_{m}$ denote the common modulation frequency and wave number, $\Delta n_{1}, \phi_{1}$, and $\Delta n_{2}, \phi_{2}$ are modulation amplitudes and phases for the real and imaginary parts, respectively. In practice, the simultaneous real and imaginary index modulation can be realized by using combined phase (or frequency) and amplitude modulators [60-62]. As shown in Fig. 1(c), the modulation of refractive index can induce photonic intraband transitions among a discrete set of optical slab modes with frequency $\omega_{n}=\omega_{0}+n \Omega$ and wave number $\beta_{n}=\beta_{0}+n q(n=0, \pm 1, \pm 2, \ldots)$, forming a synthetic frequency lattice, where $n$ is lattice site index, $\omega_{0}$ is the central frequency, and $q$ is the wave number spacing between adjacent sites. The mode amplitudes $a_{n}(z)$ at $n t h$ site of the synthetic lattice is governed by the following coupledmode equation (see Appendix A):

$$
\begin{aligned}
i \frac{\partial a_{n}(z)}{\partial z}= & {\left[C_{1} e^{i\left(\Delta q z+\phi_{1}\right)}+i C_{2} e^{i\left(\Delta q z+\phi_{2}\right)}\right] a_{n-1}(z) } \\
& +\left[C_{1} e^{-i\left(\Delta q z+\phi_{1}\right)}+i C_{2} e^{-i\left(\Delta q z+\phi_{2}\right)}\right] a_{n+1}(z),
\end{aligned}
$$
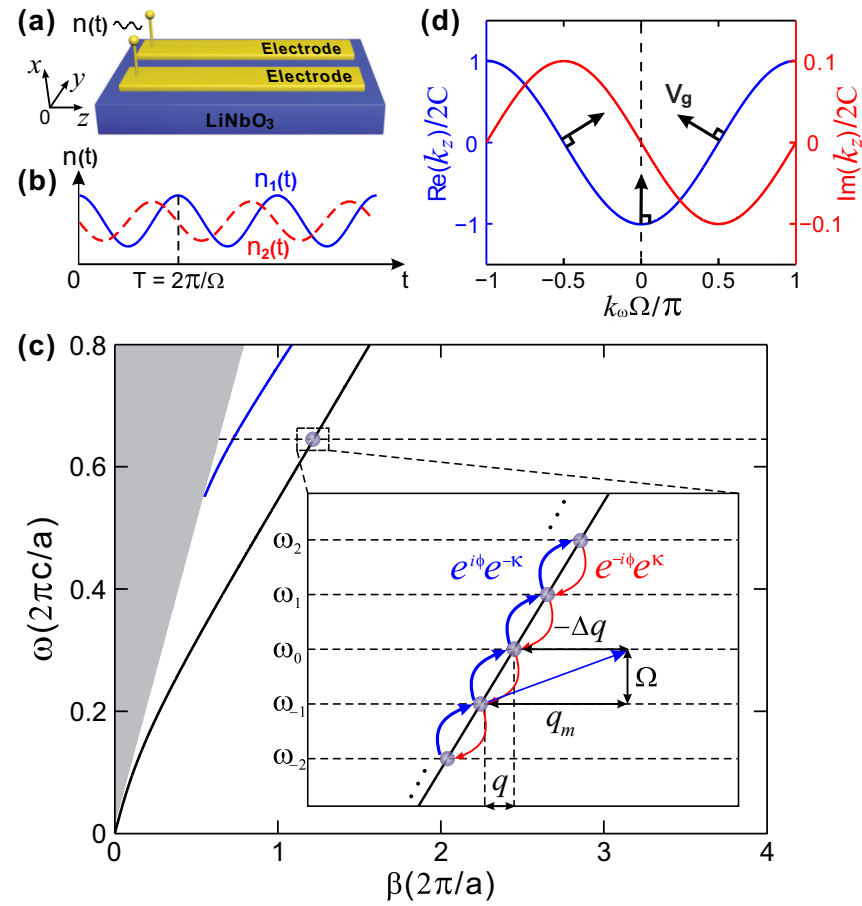

FIG. 1. (a) Schematic of a $\mathrm{LiNbO}_{3}$ waveguide modulator subject to a complex index modulation. The slab waveguide has a background refractive index $n_{0}=2.14$ and thickness $d=0.5 a$, where $a=1 \mu \mathrm{m}$. (b) The blue solid and red dashed curves denote the real $n_{1}(t)$ and imaginary $n_{2}(t)$ part of refraction index at $z=0$. (c) Dispersion curves of optical modes and schematic of photonic transitions, where the black and blue curves denote $\mathrm{TE}_{0}$ and $\mathrm{TE}_{1}$ bands and the grad region denotes the light cone. $\Omega, q_{m}$ are the modulation frequency and wave number and $q$ is the wave number spacing between adjacent modes, such that the wave number mismatch is $\Delta q=q-q_{m} . e^{i \phi} e^{-\kappa}\left(e^{-i \phi} e^{\kappa}\right)$ is the complex hopping phase during upward and downward transitions. (d) Complex band structure with the blue and red curves denoting the real and imaginary parts. Here the imaginary gauge potential is chosen as $\kappa=0.1$. The solid black arrows denote the group velocities of Bloch wave packet with $\phi_{0}-\phi=-\pi / 2,0$ and $\pi / 2$, respectively.

where $C_{1}=\Delta n_{1} k_{0} / 2, C_{2}=\Delta n_{2} k_{0} / 2$ are the mode coupling strengths between adjacent sites induced by the real and imaginary modulations, respectively, and $\Delta q=q-q_{m}$ is the wave number mismatch of the transition.

Let us first consider the perfect phase-matching regime. For $\Delta q=0$, Eq. (1) reduces to

$$
\begin{aligned}
i \frac{\partial a_{n}(z)}{\partial z}= & \left(C_{1} e^{i \phi_{1}}+i C_{2} e^{i \phi_{2}}\right) a_{n-1}(z) \\
& +\left(C_{1} e^{-i \phi_{1}}+i C_{2} e^{-i \phi_{2}}\right) a_{n+1}(z) .
\end{aligned}
$$

The Bloch modes of the frequency lattice are readily obtained making the Ansatz $a_{n}(z)=\exp \left(i n k_{\omega} \Omega\right) \exp \left(i k_{z} z\right)$, where $k_{\omega}$ is the Bloch wave number along frequency dimension and $k_{z}$ the eigenpropagation constant along $z$ direction, and

$$
k_{z}\left(k_{\omega}\right)=-2 C_{1} \cos \left(k_{\omega} \Omega-\phi_{1}\right)-2 i C_{2} \cos \left(k_{\omega} \Omega-\phi_{2}\right),
$$

is the dispersion curve of the tight-binding lattice band. Note that, contrary to most common Hermitian lattices with 
purely real band structures [3-7], the frequency lattice here is non-Hermitian with a complex band structure. In particular, with appropriate choices of real and imaginary modulation amplitudes and phases, an effective complex gauge potential is obtained in the frequency lattice. Like in the HatanoNelson model [24,25], a complex-valued gauge potential $A=$ $A_{1}+i A_{2}$ yields a complex phase factor $e^{i \int_{\omega_{n}}^{\omega_{n+1}}\left(A_{1}+i A_{2}\right) d \omega}=$ $e^{i A_{1} \Omega} e^{-A_{2} \Omega}=e^{i \phi} e^{-\kappa}$ accompanying frequency transitions, so that the coupled-mode equation takes the following form:

$$
i \frac{\partial a_{n}(z)}{\partial z}=C\left[e^{i \phi} e^{-\kappa} a_{n-1}(z)+e^{-i \phi} e^{\kappa} a_{n+1}(z)\right],
$$

where $C$ is the real-valued coupling strength in the absence of complex gauge potential. Clearly, a nonvanishing value of $\kappa$ yields asymmetric hopping in the lattice. Note that, in terms of $C$ and $\kappa$, the band structure can be written in the equivalent form

$$
\begin{aligned}
k_{z}\left(k_{\omega}\right)= & -2 C \cos \left(k_{\omega} \Omega-\phi-i \kappa\right) \\
= & -2 C \cosh (\kappa) \cos \left(k_{\omega} \Omega-\phi\right) \\
& -2 i C \sinh (\kappa) \cos \left(k_{\omega} \Omega-\phi-\pi / 2\right) .
\end{aligned}
$$

By comparing Eqs. (5) and (3), we can obtain the conditions to synthesize a complex gauge potential: $C_{2} / C_{1}=$ $\Delta n_{2} / \Delta n_{1}=\tanh (\kappa)$ and $\phi_{2}-\phi_{1}=\pi / 2$, from which we have $\kappa=\operatorname{In}\left[\left(1+\Delta n_{2} / \Delta n_{1}\right) /\left(1-\Delta n_{2} / \Delta n_{1}\right)\right] / 2$. So the imaginary gauge potential is controlled by the ratio between the imaginary and real modulation amplitudes. As illustrated in Fig. 2(a), to ensure that $\kappa$ has its physical meaning, the ratio should be chosen in the regime $0<\Delta n_{2} / \Delta n_{1}<1$, indicating that the imaginary modulation amplitude cannot overcome its real part. In particular, in the limit $\Delta n_{2} / \Delta n_{1} \rightarrow 0$, we have $\kappa \rightarrow 0$, the gauge potential becomes entirely real and the usual Hermitian discrete diffraction of frequency modes, with conserved energy and symmetric patterns, is observed [52]. On the other hand, in the opposite limit $\Delta n_{2} / \Delta n_{1} \rightarrow 1$, we have $\kappa \rightarrow \infty$, which means that the frequency transition is permitted in one direction while completely inhibited in the other. This limit corresponds to the non-Hermitian lattices with unidirectional hoppling strengths [61]. It is worth considering the asymptotic behavior of $\kappa$ in the small modulation limit $\Delta n_{2} / \Delta n_{1} \rightarrow 0$, which is of main practical relevance. In this limit one has $\kappa=\Delta n_{2} / \Delta n_{1} /\left(1-\Delta n_{2} / \Delta n_{1}\right)=$ $\Delta n_{2} / \Delta n_{1}$, i.e., the imaginary gauge potential is proportional to the ratio of imaginary to real modulation amplitudes. This feature can be clearly seen from Fig. 2(a) and in the inset figure, where the blue solid analytic curve and red dashed asymptotic line coincide well with each other. Throughout the paper, we restrict our choice of $\kappa$ in this linear regime, such that $\kappa=\Delta n_{2} / \Delta n_{1}$.

Tailoring the complex gauge potential enables to control both the frequency shift and energy evolution of the spectral modes. For a finite-width Bloch-wave packet (i.e., an optical pulse train) incident into the system, in terms of the band structure shown in Fig. 1(d), the wave packet group velocity is $v_{g, \omega}=-\partial \operatorname{Re}\left[k_{z}\left(k_{\omega}\right)\right] / \partial k_{\omega}=$ $-2 C \Omega \cosh (\kappa) \sin \left(\phi_{0}-\phi\right)$, giving rise to a total frequency shift $\Delta \omega=v_{g, \omega} L=-m_{\varphi} \Omega \cosh (\kappa) \sin \left(\phi_{0}-\phi\right)$, where $\phi_{0}=$ $k_{\omega} \Omega$ is the initial central Bloch momentum, $m_{\varphi}=\Delta n k_{0} L=$ $2 C L$ is the phase modulation depth and $L$ is the modu-
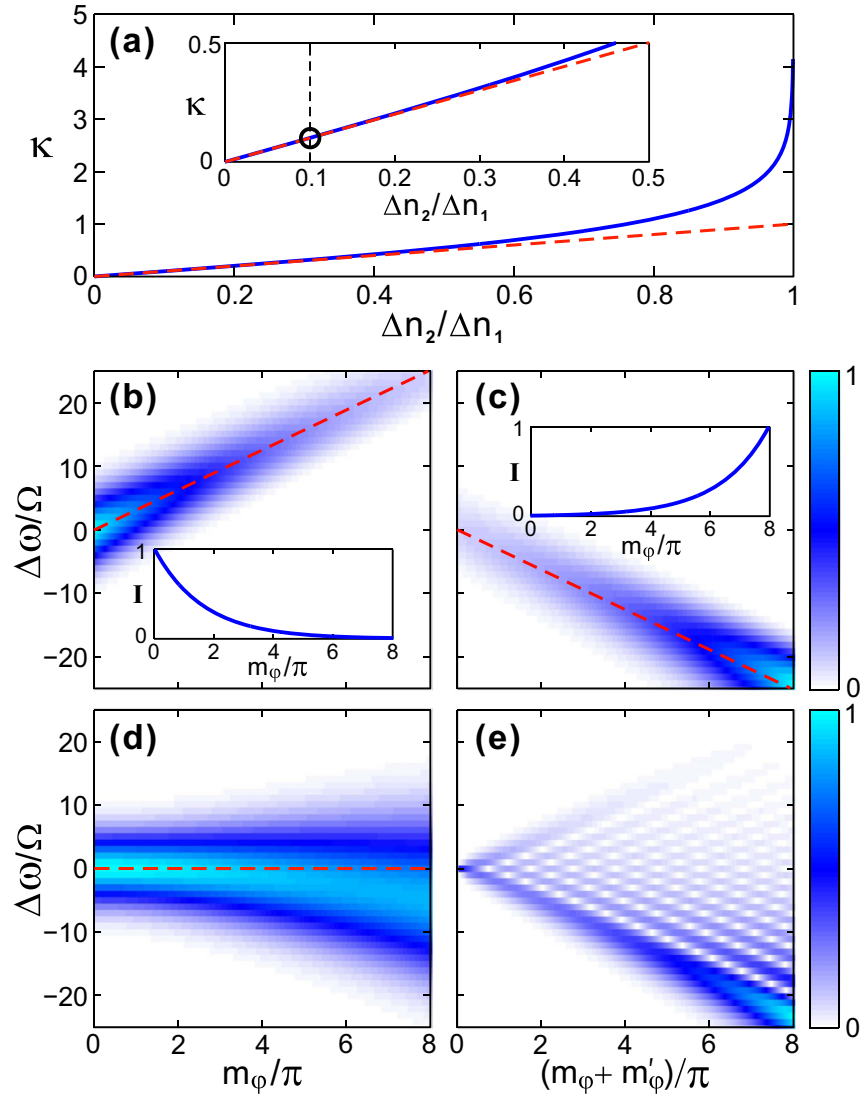

FIG. 2. (a) Imaginary gauge potential as a function of the ratio between the imaginary and real index modulation amplitudes. We choose $\kappa=0.1$ in the calculations of this figure and Fig. 3, as denoted by the black circle in the inset figure of (a). (b)-(d) Frequency discrete diffraction for the input Bloch-wave packet versus the phase modulation depth $m_{\varphi}$ in a single modulator for $\Delta q=0$. The phase modulation depth is related to the propagation distance through $m_{\varphi}(z)=\Delta n k_{0} z$. For a waveguide modulator with fixed length $L$, the accumulated phase modulation depth is $m_{\varphi}=\Delta n k_{0} L=2 C L$. So we can linearly increase the phase modulation depth to mimic the spectrum evolution with the increase of coordinate $z$. The packet initial Bloch momentum is (b) $\phi_{0}-\phi=-\pi / 2$, (c) $\phi_{0}-\phi=\pi / 2$ and (d) $\phi_{0}-\phi=0$. The inset figures in (b) and (c) denote the packet energy evolutions. (e) Spectrum evolution for a single frequency input in a single modulator, manifesting the asymmetric discrete diffraction.

lator length. The wave packet energy evolves as $I(z)=$ $\left|a_{n}(z)\right|^{2}=I_{0} \exp \left[-2 \operatorname{Im}\left(k_{z}\right) z\right]$, which reaches the value $I(L)=$ $I_{0} \exp \left[2 m_{\varphi} \sinh (\kappa) \sin \left(\phi_{0}-\phi\right)\right]$ at the output end $z=L$, where $I_{0}$ is the initial intensity. So at the right-half part of Brillouin zone $0<\phi_{0}-\phi<\pi$, we have $\Delta \omega<0, I(L)>I_{0}$, the packet experiences a red spectral shift accompanied by an exponential energy growth. On the contrary, at the left-half part $-\pi<\phi_{0}-\phi<0, \Delta \omega>0, I(L)<I_{0}$, the wave packet exhibits a spectrum blue shift with an exponential energy decay. At Brillouin zone center or edges $\phi_{0}-\phi=0$ or $\pi$, $\Delta \omega=0, I(L)=I_{0}$, both frequency shift and energy do not change.

To check the validity of the theoretical analysis, we simulate spectrum dynamics by numerically solving Eq. (2). 
Throughout the paper, we choose $n_{0}=2.14$ and waveguide width $d=0.5 \mu \mathrm{m}$. The central frequency is $\omega_{0}=$ $0.6451(2 \pi c / a)$ with $a=1 \mu \mathrm{m}$, corresponding to the telecommunication wavelength $\lambda_{0}=1.55 \mu \mathrm{m}$. The modulation frequency is $\Omega / 2 \pi=10 \mathrm{GHz}$ and the modulation wave number is $q_{m}=q=0.93 \mathrm{~mm}^{-1}$ at $\omega_{0}$. The dielectric modulation amplitude is $\Delta \varepsilon=2 n_{0} \Delta n=2 \times 10^{-3}$, such that the real and imaginary parts are $\Delta \varepsilon_{1}=\Delta \varepsilon \cosh (\kappa)$ and $\Delta \varepsilon_{2}=$ $\Delta \varepsilon \cosh (\kappa)$, respectively. Figures 2(b)-2(d) show the spectrum evolution for an input Bloch-wave packet $a_{n}(0)=$ $a_{0} \exp \left[-(n \Omega / W)^{2}\right] \exp \left(i n \phi_{0}\right)$, where $W=5 \Omega$ is the width of Gaussian envelope and $\phi_{0}$ is the initial Bloch momentum. The simulated intensity evolutions are shown in the inset figures, which are obtained through $I=\sum_{n}\left|a_{n}\right|^{2}$. In Fig. 2(b), we choose $\phi_{0}-\phi=-\pi / 2$ and $\kappa=0.1$, the packet manifests a directional frequency blue shift accompanied by an exponential energy decay. On the contrary, for $\phi_{0}-\phi=\pi / 2$ shown in Fig. 2(c), the packet exhibits a maximum spectrum red shift with an exponential intensity growth. For $\phi_{0}-\phi=0$ in Fig. 2(d), the wave packet center exhibits no spectral shift and the energy is also conversed. Note that the spectrum envelope exhibits an appreciable red shift, which is attributed to the nonvanishing momentum distribution around $\phi_{0}-\phi=$ 0 due to the finite width of the packet envelope. In Fig. 2(e), we excite the central-frequency mode in the modulator with $a_{n}(0)=a_{0} \delta_{n, 0}$, the spectrum of which manifests the characteristic cone-like discrete diffraction but with an asymmetric diffraction pattern $\left|a_{n}\right|=a_{0}\left|J_{n}\left(m_{\varphi}\right)\right| e^{-n \kappa}$ [see Eq. (B2) in Appendix B]. So compared to the traditional discrete diffraction without gauge potential or with a purely real gauge potential where the diffraction pattern is symmetric $\left|a_{n}\right|=a_{0}\left|J_{n}\left(m_{\varphi}\right)\right|$ [52], the presence of imaginary gauge potential imprints an amplified or decayed exponent on each order, giving rise to the asymmetric diffraction pattern.

Furthermore, if we cascade two modulators with different complex gauge potentials $\phi+i \kappa$ and $\phi^{\prime}+i \kappa^{\prime}$, a frequency analog of refraction will also occur, where both of the frequency shift and energy evolution can be altered at the boundary. With all combinations of complex gauge potentials taken into account, there are totally four kinds of refraction scenarios: (1) $\phi=\phi^{\prime}$ and $\kappa=\kappa^{\prime}$; (2) $\phi=\phi^{\prime}$ and $\kappa \neq \kappa^{\prime}$; (3) $\phi \neq \phi^{\prime}$ and $\kappa=\kappa^{\prime}$; (4) $\phi \neq \phi^{\prime}$ and $\kappa \neq \kappa^{\prime}$. In all scenarios, the refraction for a Bloch-wave packet can be analyzed from band structure perspective. Suppose the packet carries an initial Bloch momentum $\phi_{0}=k_{\omega} \Omega$, we can readily define an effective relative refractive index between the two modulators

$$
n_{r, \mathrm{eff}}=\frac{k_{z, 1}\left(k_{\omega}\right)}{k_{z, 2}\left(k_{\omega}\right)}=\frac{\cos \left(\phi_{0}-\phi-i \kappa\right)}{\cos \left(\phi_{0}-\phi^{\prime}-i \kappa^{\prime}\right)} .
$$

So under different combinations of complex gauge potentials, we can achieve versatile refraction phenomena, including both positive and negative spectrum or energy refractions. Additionally, when we consider a single frequency input, the band structure analysis is invalid: an input single frequency contains all Bloch-mode components in the Brillouin zone, making the definition of effective relative refractive index invalid. Instead, the refraction can be described by the refraction patterns calculated from optical phase modulation. The detailed theoretical analysis is provided in Appendix B.
We start from case (1) by choosing $\phi_{0}-\phi=\phi_{0}-\phi^{\prime}=$ $\pi / 2$ and $\kappa=\kappa^{\prime}=0.1$. The two modulators share the same band structures shown in Fig. 3(a). The relative refractive index is thus $n_{r, \text { eff }}=1$, indicating that the refraction will vanish and degrade into the diffraction in a single modulator. In Figs. 3(b) and 3(c), we input at a Bloch-wave packet and a single frequency into the system, both of which share the same diffraction patterns with those in Figs. 2(b) and 2(e). Specifically, the refraction pattern in Fig. 3(c) is theoretically described by $\left|a_{n}\right|=a_{0}\left|J_{n}\left(m_{\varphi}+m_{\varphi}^{\prime}\right)\right| e^{-n \kappa}$ [see Eq. (B5) in Appendix B], where $m_{\varphi}, m_{\varphi}^{\prime}$ are phase modulation depths in the two modulators.

Then, we consider case (2) by choosing $\phi_{0}-\phi=\phi_{0}-$ $\phi^{\prime}=\pi / 2$ and $\kappa=-\kappa^{\prime}=0.1$. As shown in Fig. 3(d), the real part of band structure in the second modulator is the same with that of the first one, while the imaginary part is flipped, giving rise to a negative relative refractive index $n_{r, \text { eff }}=-1$. As a result, the refraction direction keeps unchanged but the energy evolution will be reversed, which can be referred to as the "energy negative refraction". This process can be visualized in Fig. 3(e), where the packet exhibits a directional spectral blue shift all through the way accompanied by the energy decay in first modulator and then growth in the second, ultimately restoring to its input intensity at the output end. For a single frequency input shown in Fig. 3(f), due to the reverse of $\kappa$, the refraction pattern is biased in lower frequency modes in first modulator and then biased in higher frequency modes in the second, ultimately giving rise a symmetric output spectrum $\left|a_{n}\right|=a_{0}\left|J_{n}\left[2 m_{\varphi} \cosh (\kappa)\right]\right|$. [See Eq. (B8) in Appendix B]. In this regard, the energy negative refraction can restore the energy but cannot restore the spectrum distribution.

Next, we turn to case (3) for $\phi_{0}-\phi=-\pi / 2, \phi_{0}-\phi^{\prime}=$ $\pi / 2$ and $\kappa=\kappa^{\prime}=0.1$. The band structures are shown in Fig. 3(g), where the phase shift $\phi^{\prime}-\phi=\pi$ gives rise to both the real-part shift and imaginary-part flip of the band structure, such that $n_{r \text {,eff }}=-1$. So the input packet will exhibit a negative refraction where both the refraction direction and energy evolution are flipped at the boundary, as shown in Fig. 3(h). This situation is referred to as "frequency-energy negative refraction". For a single frequency input shown in Fig. 3(i), it exhibits asymmetric diffraction in the first modulator and then a time-reversal process in the second, ultimately focusing perfectly onto a single frequency at the output end. This process can be regarded as frequency-energy perfect imaging with an asymmetric imaging pattern $\left|a_{n}\right|=$ $a_{0}\left|J_{n}\left(m_{\varphi}-m_{\varphi}^{\prime}\right)\right| e^{-n \kappa}$ [see Eq. (B5) in Appendix B]. This frequency-energy negative refraction and perfect imaging are reminiscent of spatial superlens made of $n=-1$ slab [63], which can realize the perfect imaging of a point light source by reversing all wave number components. In practice, the capability of simultaneous spectrum and energy restoration may find applications in spectral imaging and reconstruction for the purposes of signal processing and optical communications [64-70].

Finally, we investigate case (4) with $\phi_{0}-\phi=-\pi / 2, \phi_{0}-$ $\phi^{\prime}=\pi / 2$ and $\kappa=-\kappa^{\prime}=0.1$. The band structures are shown in Fig. 3(j), where the real parts are opposite to each other while the imaginary parts are the same, giving rise to also a negative relative refractive index $n_{r \text {,eff }}=-1$. As shown in Fig. 3(k), the refraction direction is reversed at the boundary 


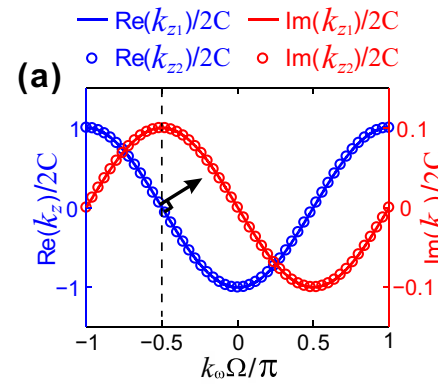

(b)

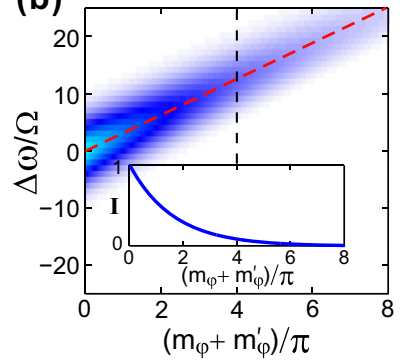

(c)

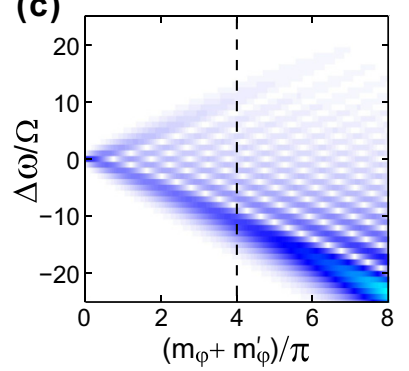

(d)

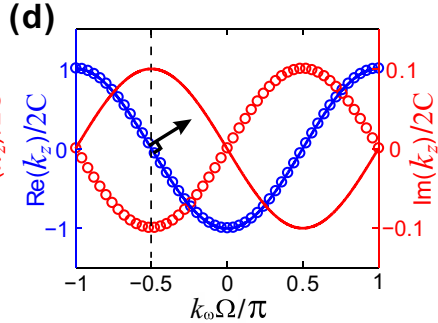

(e)

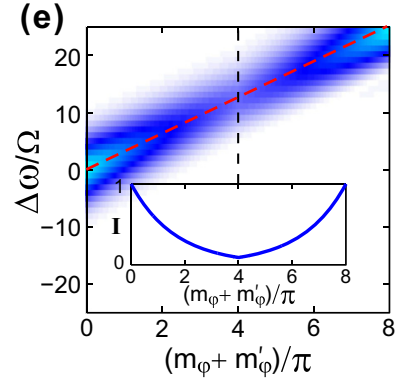

(f)

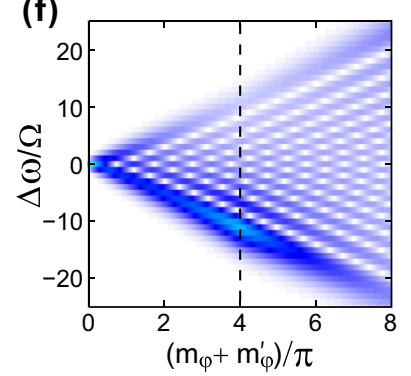

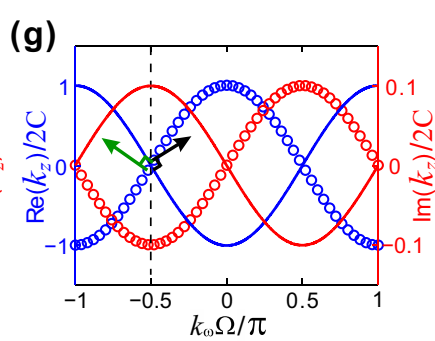

(h)

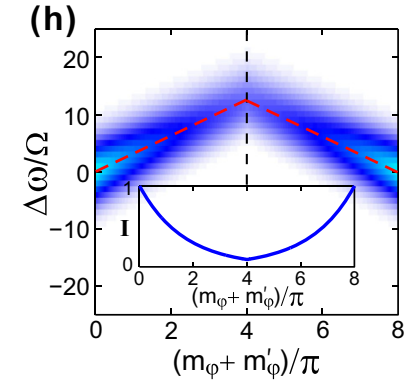

(i)

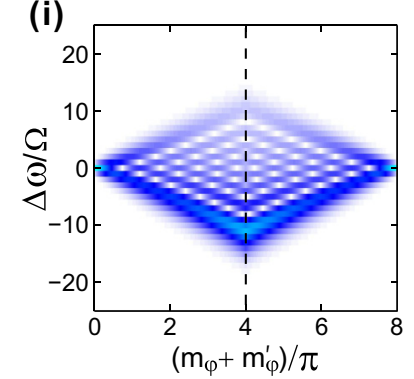

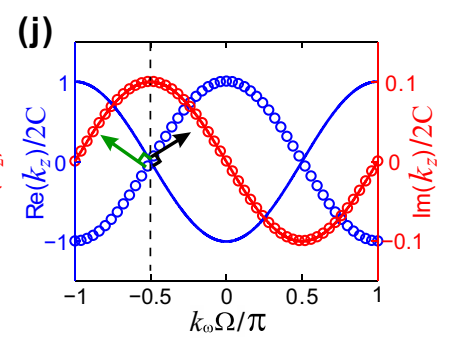

(k)
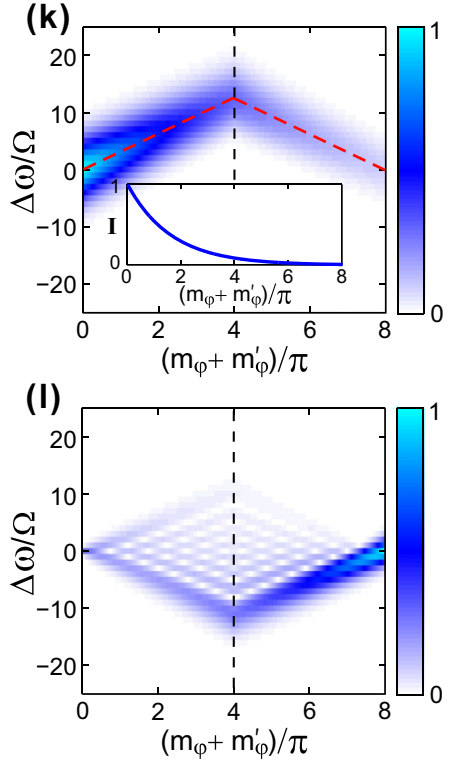

FIG. 3. (a),(d),(g),(i) Real and imaginary parts of the lattice band structures in the two modulators with (a) $\phi=\phi^{\prime}=0$ and $\kappa=\kappa^{\prime}=0.1$, (d) $\phi=\phi^{\prime}=0$ and $\kappa=-\kappa^{\prime}=0.1$, (g) $\phi=0, \phi^{\prime}=\pi$ and $\kappa=\kappa^{\prime}=0.1$, (i) $\phi=0, \phi^{\prime}=\pi$ and $\kappa=-\kappa^{\prime}=0.1$. The black dashes lines denote the initial Bloch momentum carried by the input packet. The black and green arrows denote the group velocities in the frequency lattices of two modulators. (b),(e),(h),(k) Frequency refraction for an input Bloch-wave packet versus the total modulation depth $m_{\varphi}+m_{\varphi}^{\prime}$, where $m_{\varphi}$ and $m_{\varphi}^{\prime}$ are the modulation depth in each modulator. The initial Bloch momentum is (b) $\phi_{0}-\phi=\phi_{0}-\phi^{\prime}=-\pi / 2$, (e) $\phi_{0}-\phi=\phi_{0}-\phi^{\prime}=-\pi / 2$, (h) $\phi_{0}-\phi=-\pi / 2$ and $\phi_{0}-\phi^{\prime}=\pi / 2$, (k) $\phi_{0}-\phi=-\pi / 2, \phi_{0}-\phi^{\prime}=\pi / 2$. The red dashes lines in (b),(e),(h),(k) represent the packet-center evolution trajectories, and the inset figures denote the packet energy evolutions. (c),(f),(i),(l) Spectrum evolutions for a single frequency input with the parameters chosen with the same in (a),(d),(g),(i). The black dashed lines in (b),(e),(h),(k) and (c),(f),(i),(l) denote the boundaries of the two modulators.

while the energy decay is kept, which can be referred to as "frequency negative refraction". In Fig. 3(1), we input a single frequency into the system, where the refraction pattern is given by Eq. (B11) in Appendix B. Due to the reverse of $\kappa$, it cannot be focused onto itself since the energy cannot be focused, in stark contrast to the situation in Fig. 3(i). To sum up, though cases (2)-(4) all give rise to a negative refractive index $n_{r \text {,eff }}=-1$, the results vary with one another: while case (2) realizes only energy negative refraction and case (4) achieves purely frequency negative refraction, case (3) can realize both frequency and energy negative refraction and imaging. All of these refraction effects could be useful for both spectrum and energy control of light.

\section{B. Non-Hermitian frequency-domain Bloch oscillations}

Bloch oscillations in the non-Hermitian frequency lattice can also arise when considering the phase-mismatching case $\Delta q \neq 0$, which is equivalent to a gradient force applied in the frequency space [12,59]. The $z$-dependent coupled-mode equations for in the phase-mismatched regime read

$$
i \frac{\partial a_{n}(z)}{\partial z}=C\left[e^{i\left(\Delta q z+\phi_{1}+i \kappa\right)} a_{n-1}(z)+e^{-i\left(\Delta q z+\phi_{1}+i \kappa\right)} a_{n+1}(z)\right],
$$

corresponding to the instantaneous band structure

$$
\begin{aligned}
k_{z}\left[k_{\omega}(z)\right]= & -2 C \cos \left[k_{\omega}(z) \Omega-\phi-i \kappa\right] \\
= & -2 C \cosh (\kappa) \cos \left[k_{\omega}(z) \Omega-\phi\right] \\
& -2 i C \sinh (\kappa) \cos \left[k_{\omega}(z) \Omega-\phi-\pi / 2\right] .
\end{aligned}
$$

Comparing Eqs. (8) and (5), one sees that the band structure remains functionally unchanged, however the Bloch wave vector $k$ varies linearly with $z$ as $k_{\omega}(z)=k_{\omega}-\Delta q z / \Omega$ as if an effective electric force $F_{1}=-\Delta q$ is impressed to the system. According to the well-known "acceleration theorem," for a Bloch-wave packet input excitation, the instantaneous group velocity is $v_{g, \omega}(z)=-\partial \operatorname{Re}\left\{k_{z}\left[k_{\omega}(z)\right]\right\} / \partial k_{\omega}(z)=$ $-2 C \Omega \cosh (\kappa) \sin \left(\phi_{0}-\phi-\Delta q z\right)$, corresponding to a frequency shift of $\Delta \omega(z)=\int_{0}^{z} v_{g, \omega}\left(z^{\prime}\right) d z^{\prime}$, so the frequency- 

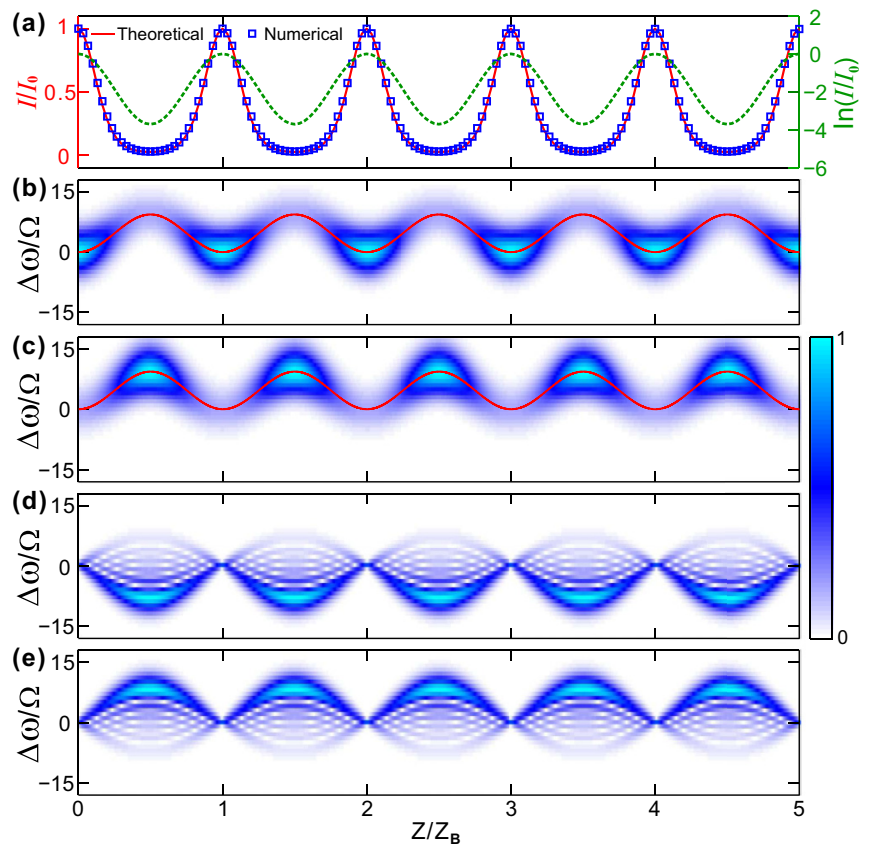

FIG. 4. (a) Intensity oscillation trajectory of a Bloch-wave packet versus the normalized propagation distance $z / Z_{\mathrm{B}}$ during nonHermitian Bloch oscillations with $\phi_{0}-\phi=\pi$ and $\kappa=0.2$. Here $Z_{\mathrm{B}}$ is the spatial period of Bloch oscillations. The red solid and blue rectangular curves represent the theoretical and numerical trajectories with the green dashed curve denoting the exponential intensity evolution. (b), (c) Packet spectrum evolutions $\left|a_{n}\right|$ with $\phi_{0}-\phi=\pi$ for (b) $\kappa=0.2$, (c) $\kappa=-0.2$. (d), (e) Single frequency evolutions for (d) $\kappa=0.2$, (e) $\kappa=-0.2$.

domain oscillation trajectory is

$\Delta \omega(z)=\frac{2 C \Omega \cosh (\kappa)}{\Delta q}\left[\cos \left(\phi-\phi_{0}\right)-\cos \left(\Delta q z+\phi-\phi_{0}\right)\right]$.

Meanwhile, the packet intensity evolves according to $\partial \operatorname{In}[I(z)] / \partial z=-2 \operatorname{Im}\left\{k_{z}\left[k_{\omega}(z)\right]\right\}$ [26], from which we have $I(z)=I_{0} \exp \left(-\int_{0}^{z} 2 \operatorname{Im}\left\{k_{z}\left[k_{\omega}\left(z^{\prime}\right)\right]\right\} d z^{\prime}\right)$, which reads

$I(z)=I_{0} \exp \left\{\frac{4 C \sinh (\kappa)}{\Delta q}\left[\cos \left(\Delta q z+\phi-\phi_{0}\right)-\cos \left(\phi-\phi_{0}\right)\right]\right\}$

As compared to traditional Bloch oscillations with constant packet energy, the presence of imaginary gauge potential can also induce the periodic oscillation of packet total energy [59], which is a typical signature of the non-Hermitian Bloch oscillations. Note that both the packet center-of-mass and total energy share the same oscillation period of $Z_{\mathrm{B}}=2 \pi /\left|F_{1}\right|=$ $2 \pi /|\Delta q|$.

To visualize the non-Hermitian Bloch oscillations with periodic intensity evolution, we perform numerical simulations by solving Eq. (1). Figure 4(a) shows the intensity evolution of a Bloch-wave packet with $\phi_{0}-\phi=\pi, \kappa=0.2$, where $q_{m}=2 q$ such that $\Delta q=-q$. The simulated intensity evolution (blue rectangular curve) agrees well with the theoretical result $I(z)=I_{0} \exp \{4 C \sinh (\kappa)[1-\cos (\Delta q z)] / \Delta q\}$ (red solid curve). The intensity reaches $I_{\max }=I_{0}$ at integer periods $z=$ $m Z_{\mathrm{B}}$ and $I_{\min }=I_{0} \exp [4 C \sinh (\kappa) / \Delta q]$ at half integer periods $z=(m+1 / 2) Z_{\mathrm{B}}$, where $m$ is an integer. The exponential intensity evolution is also shown as the dashed green curve, which manifests a cosine oscillation trajectory, also in good agreement with theoretical prediction by Eq. (10). Figure 4(b) shows the packet spectrum evolution, which exhibits both oscillatory frequency shift and energy distribution. In Fig. 4(c), we flip $\kappa=0.2$ to $\kappa=-0.2$, the spectrum oscillation trajectory remains unchanged while the energy oscillation trajectory is shifted by half a period. It is attributed to the fact that the oscillation trajectory is independent of the sign of imaginary gauge potential as the intensity evolution does according to Eqs. (9) and (10). In Figs. 4(d) and 4(e), we input a single frequency $\omega_{0}$ instead of a wave-packet into the system and choose $\kappa=0.2$ and $\kappa=-0.2$. In both cases, the single frequency manifests periodic "self-focusing" features, showing asymmetric "breathing" patterns described by $\left|a_{n}\right|=$ $a_{0}\left|J_{n}[4 C \sin (\Delta q z / 2) / \Delta q]\right| e^{-n \kappa}$ [9]. The total energy is more concentrated in low-frequency modes for $\kappa>0$ and in highfrequency modes for $\kappa<0$.

The above asymmetric behavior of the breathing dynamics for a single frequency excitation is a clear bulk signature of the NHSE associated to the single-band non-Hermitian lattice with an imaginary gauge potential, i.e., with asymmetric hopping rates. We will show in the following that the breathing dynamics is always symmetric in the absence of the NHSE, where the frequency center is locked to the input frequency, while it becomes asymmetric when the system shows the NHSE. This means that non-Hermitian Bloch oscillations can provide a probing method in the bulk to detect the existence (or not) of the NHSE in the lattice with OBCs [26-44]. Without loss of generality, let us consider the generalized coupled-mode equation for a non-Hermitian lattice with longrange couplings and a gradient force

$$
i \frac{\partial a_{n}(z)}{\partial z}=\sum_{l}\left[C_{l} a_{n-l}(z)+C_{-l} a_{n+l}(z)\right]+F n a_{n}(z),
$$

where $C_{l}=C_{l, 0} e^{i l \phi} e^{-l \kappa}$ and $C_{-l}=C_{l, 0} e^{-i l \phi} e^{l \kappa}(l=1,2, \ldots)$ are $l$ th-order long-range coupling coefficients for the up and downward transitions between lattice sites with frequency spacing $l \Omega . C_{l, 0}$ is the coupling coefficient without gauge potentials. $F$ is the gradient force applied to generate Bloch oscillations. As the force term vanishes $F=0$, the lattice band structure reads

$$
k_{z}\left(k_{\omega}\right)=-\sum_{l} C_{l, 0} \exp \left[-i l\left(k_{\omega} \Omega-\phi-i \kappa\right)\right] .
$$

So in the absence of complex gauge potential $\phi=\kappa=$ 0 , the coupling coefficients satisfy the symmetric condition $C_{l}=C_{-l}=C_{l, 0}$, the curve $k_{z}\left(k_{\omega}\right)$ will describe an open arc in the complex energy plane without any interior. In this case, the NHSE will not be observed [26-44], the bulk energy spectrum is thus independent of the boundary conditions and the bulk states are extended over the entire lattice. Note that the symmetric condition corresponds to $k_{z}\left(k_{\omega}\right)=k_{z}\left(-k_{\omega}\right)$, which in the Hermitian limit states the time-reversal symmetry of the lattice. On the contrary, in the presence of gauge potential $\phi, \kappa \neq 0$, the coupling coefficients become asymmetric $C_{l} \neq C_{-l}$. The curve $k_{z}\left(k_{\omega}\right)$ will trace out a closed loop in 

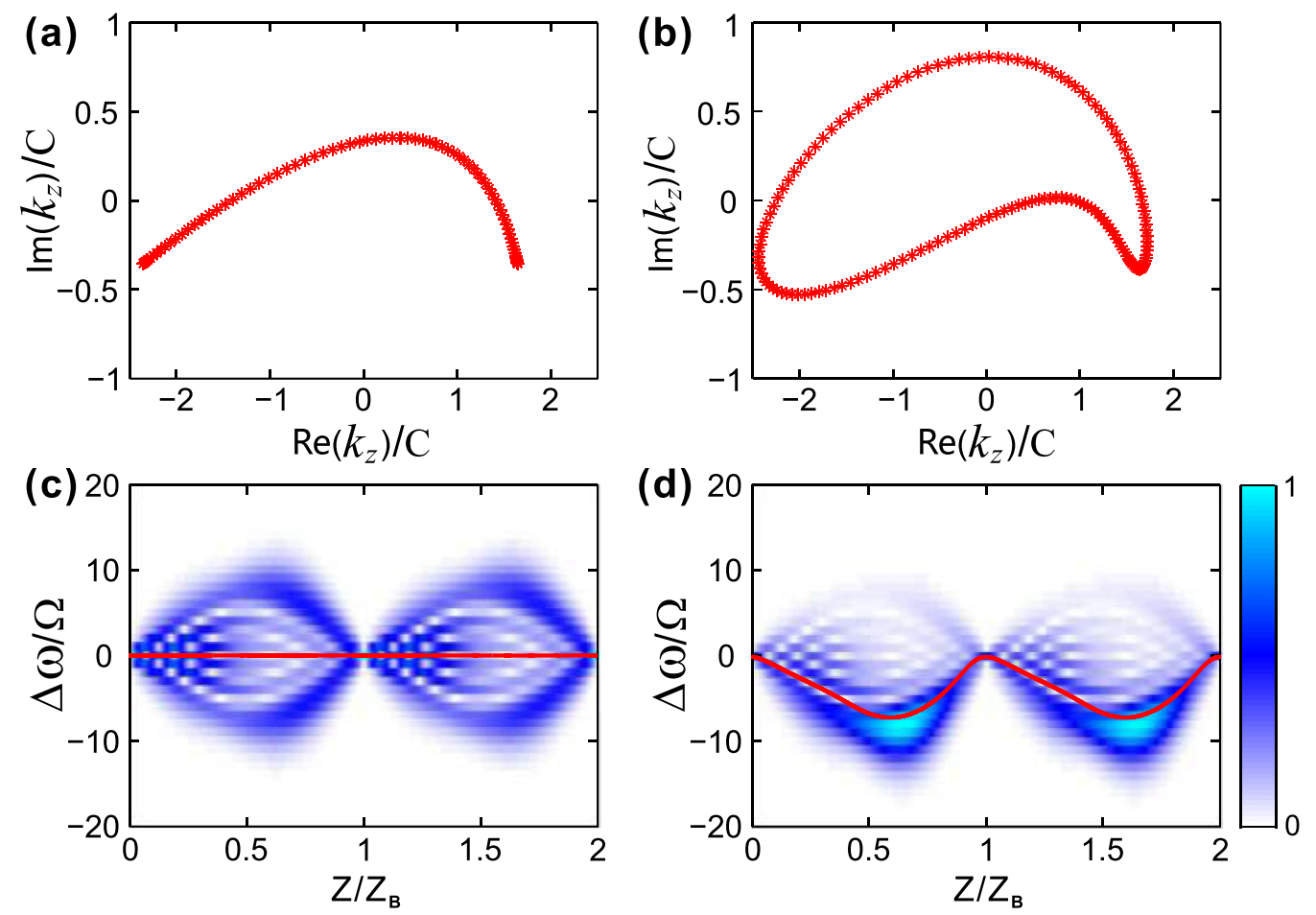

FIG. 5. (a)(b) Energy spectra in the complex plane defined by Eq. (12) with $C_{2,0}=C_{1,0} \exp (i \pi / 4) / 4$ and $C_{1,0}=C$ for (a) $\phi=\kappa=0$, (b) $\phi=0, \kappa=0.2$. (c),(d) Spectrum evolutions for a single frequency excitation corresponding the situations of (a) and (b). The red solid curves denote the frequency center-of-mass calculated using Eq. (15). The magnitude of the force is chosen as $F=|\Delta q|$.

the complex energy plane with finite interior, i.e., enclosing a nonvanishing area. In this case, the lattice can manifest the NHSE where the bulk states are squeezed toward the edges when considering OBCs. Examples of complex energy spectra corresponding to open arc and closed loop are shown in Figs. 5(a) and 5(b), where only the nearest-neighbor $l=1$ and next-to-nearest neighbor couplings $l=2$ are included. In Fig. 5(a), we choose $\phi=\kappa=0, C_{2,0}=C_{1,0} \exp (i \pi / 4) / 4$ and $C_{1,0}=C$, the energy spectrum in the complex plane describes an open arc. In contrast, with a nonvanishing imaginary gauge potential $\kappa=0.2$ in Fig. 5(b), the energy spectrum traces out a closed loop in the complex plane. In this regard, the complex gauge potential can be engineered to control the energy spectrum shape and hence the emergence or not the NHSE. Let us then consider the case of nonvanishing external force $F$. As shown in previous works [59], the energy spectrum is entirely real and given by the Wannier-Stark ladder $E_{l}=$ $l F(l=0, \pm 1, \pm 2, \ldots)$. Hence, the dynamics are oscillatory with period $2 \pi /|F|$, whether the system does or does not show the NHSE. To probe the NHSE in the bulk, let us consider the Bloch oscillation breathing dynamics that arises from a single cite excitation $a_{n}(0)=a_{0} \delta_{n, 0}$, corresponding in our model of single frequency excitation. The exact analytical solution to Eq. (11) can be determined and reads [59]

$$
a_{n}(z)=a_{0} \frac{\exp (-i F n z)}{2 \pi} \int_{-\pi / \Omega}^{\pi / \Omega} \exp \left\{i k_{\omega} \Omega n-i G\left(k_{\omega}, z\right)\right\} d k_{\omega},
$$

where we have set

$$
G\left(k_{\omega}, z\right)=\int_{0}^{z} k_{z}\left(k_{\omega} \Omega-F z^{\prime}\right) d z^{\prime}
$$

The asymmetry of the breathing dynamics can thus be revealed by computing the center-of-mass of the spectral pattern $\langle\Delta \omega(z) / \Omega\rangle$ versus $z$, which is given by

$$
\left\langle\frac{\Delta \omega(z)}{\Omega}\right\rangle=\frac{\sum_{n} n\left|a_{n}(z)\right|^{2}}{\sum_{n}\left|a_{n}(z)\right|^{2}}
$$

Remarkably, as the coupling coefficients are symmetric, i.e., when the system does not show the NHSE, one has $\langle\Delta \omega(z) / \Omega\rangle=0$. The mean frequency thus remains locked at the excitation frequency, as clearly shown by the red solid curve in Fig. 5(c). Such a property readily follows from the fact that, provided that $k_{z}\left(-k_{\omega}\right)=k_{z}\left(k_{\omega}\right)$, one has $G\left(-k_{\omega}\right)=$ $G\left(k_{\omega}\right)$ as well, which gives rise to $\left|a_{-n}(z)\right|=\left|a_{n}(z)\right|$. On the other hand, for the asymmetric coupling coefficients, the breathing dynamics become asymmetric $\left|a_{-n}(z)\right| \neq\left|a_{n}(z)\right|$, where $\langle\Delta \omega(z) / \Omega\rangle$ deviates from zero and undergoes an oscillatory motion shown by the red curve in Fig. 5(d). So the symmetry of the breathing pattern or center-of-mass trajectory can be used as a probing signature in the bulk to predict the presence or not the NHSE.

\section{Amplified non-Hermitian frequency-domain Bloch oscillations}

The above discussions focus on imaginary gauge potential while the electric force itself is real. A question naturally arises: What if the force itself is complex? To answer this question, we generalize $F_{1}=-\Delta q$ to $F=F_{1}+i F_{2}$, and 
rewrite the lattice band structure from Eq. (8) as

$$
\begin{aligned}
k_{z}\left[k_{\omega}(z)\right]= & -2 C \cos \left[k_{\omega} \Omega-\phi-\left(F_{1}+i F_{2}\right) z\right] \\
= & -2 C \cosh \left(F_{2} z\right) \cos \left(k_{\omega} \Omega-\phi-F_{1} z\right) \\
& -i 2 C \sinh \left(F_{2} z\right) \cos \left(k_{\omega} \Omega-\phi-F_{1} z-\pi / 2\right),
\end{aligned}
$$

where the gauge potential here is assumed to be real $(\kappa=0)$ for simplicity. The group velocity is $v_{g, \omega}(z)=$ $-2 C \Omega \cosh \left(F_{2} z\right) \sin \left(\phi_{0}-\phi-F_{1} z\right)$, leading to the frequency shift (See Appendix C)

$$
\begin{aligned}
\Delta \omega(z)= & \frac{2 C \Omega}{F_{1}^{2}+F_{2}^{2}}\left[F_{1} \cos \left(\phi-\phi_{0}\right)-F_{1} \cosh \left(F_{2} z\right) \cos \left(\phi-\phi_{0}\right.\right. \\
& \left.\left.+F_{1} z\right)+F_{2} \sinh \left(F_{2} z\right) \sin \left(\phi-\phi_{0}+F_{1} z\right)\right] .
\end{aligned}
$$

Similarly, the intensity evolution is $I(z)=I_{0} \exp [\alpha(z)]$, with $\alpha(z)$ being (See Appendix C)

$$
\begin{aligned}
\alpha(z)= & \frac{4 C}{F_{1}^{2}+F_{2}^{2}}\left[F_{1} \sinh \left(F_{2} z\right) \cos \left(\phi_{0}-\phi-F_{1} z\right)\right. \\
& \left.+F_{2} \cosh \left(F_{2} z\right) \sin \left(\phi_{0}-\phi-F_{1} z\right)-F_{2} \sin \left(\phi_{0}-\phi\right)\right] .
\end{aligned}
$$

Usually for weak imaginary force $F_{2} \ll F_{1}$, Eqs. (17) and (18) can reduce to

$$
\begin{aligned}
\Delta \omega(z) & =\frac{2 C \Omega}{F_{1}}\left[\cos \left(\phi-\phi_{0}\right)-\cosh \left(F_{2} z\right) \cos \left(\phi-\phi_{0}+F_{1} z\right)\right], \\
I(z) & =I_{0} \exp \left(\frac{4 C \sinh \left(F_{2} z\right)}{F_{1}} \cos \left(\phi_{0}-\phi-F_{1} z\right)\right) .
\end{aligned}
$$

So both frequency shift and light intensity exhibit oscillations with exponentially growing oscillation amplitudes, which we refer to as amplified non-Hermitian frequencydomain Bloch oscillations. The amplitudes of the oscillation trajectory and exponential light intensity are given by

$$
A_{\mathrm{B}}(z)=\frac{2 C \Omega \cosh \left(F_{2} z\right)}{\left|F_{1}\right|}, \quad A_{\operatorname{In}(I)}(z)=\frac{4 C \sinh \left(F_{2} z\right)}{\left|F_{1}\right|} .
$$

To validate the above theoretical analysis, we performed numerical simulations by choosing $F_{2}=0.05 F_{1}$. Figures 6 (a) and 6(b) show the oscillatory trajectory and exponential intensity evolution for a Bloch-wave packet. The numerical results (blue circular and square curves) agree well with the theoretical ones (red solid curves) predicted by Eq. (19). Figure 6(c) depicts the wave packet spectrum evolution, clearly showing an oscillatory motion with an exponentially growing amplitude and huge energy amplification of the optical signal during propagation. In Fig. 6(d), we input the central frequency $\omega_{0}$ into the modulator, which exhibits a breathing oscillation pattern also accompanied by energy amplification. Differing from either the symmetric "self-focusing" breathing patterns in traditional Bloch oscillations [9,53] or the asymmetric "self-focusing" patterns in above non-Hermitian Bloch oscillations shown in Fig. 4, the pattern periodicity is broken by the imaginary force, and the single frequency can no longer be focused onto itself. Instead, the spectrum focusing position is biased, for which the bandwidth is also expanded.

Finally, we propose a concrete modulator structure to realize the complex force. Comparing Eqs. (8) and (16),
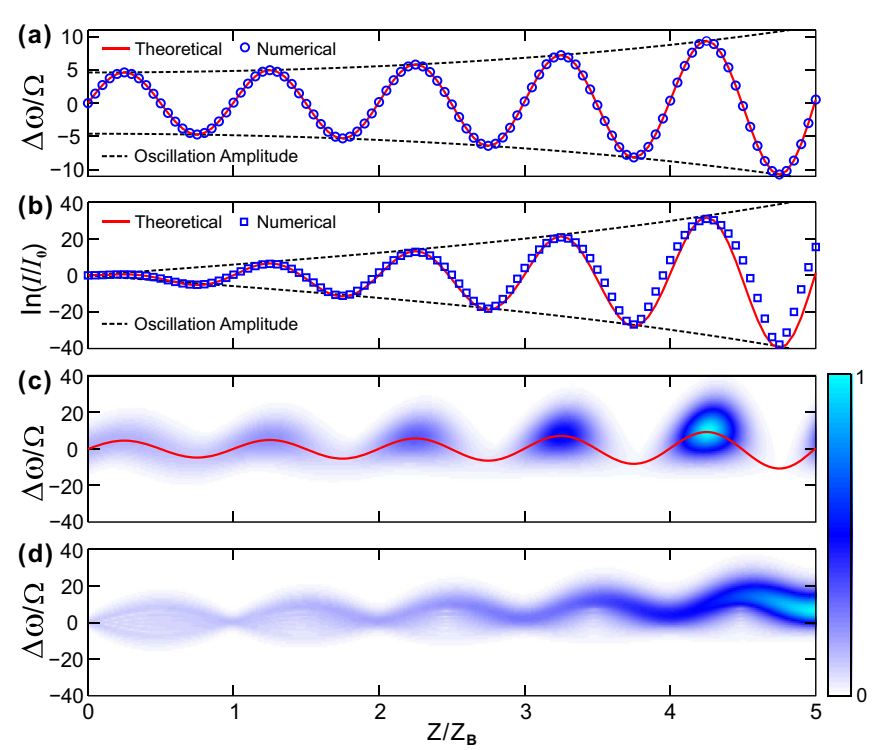

FIG. 6. (a) Oscillation trajectory of a Bloch-wave packet with $\phi_{0}-\phi=-\pi / 2$ and $F_{2}=0.05 F_{1}$. (b) The packet intensity oscillation trajectory. The red solid and blue circle (rectangular) curves represent the theoretical and numerical trajectories, and the dashed black curves denote the oscillation amplitudes. (c) The packet spectrum evolution, where the red solid curve represents the oscillation trajectory. To better visualize the energy evolution, we plot here $\left|a_{n}(z)\right|^{1 / 4}$ instead of $\left|a_{n}(z)\right|^{2}$. (d) Spectral evolution under initial single frequency excitation for $F_{2}=0.05 F_{1}$.

the imaginary force can be created by replacing the imaginary gauge potential with a linear-varying exponent. So the required complex index modulation should take the following form $n(z, t)=n_{0}+\Delta n_{1}(z) \cos \left(\Omega t-q_{m} z+\phi_{1}\right)+$ $i \Delta n_{2}(z) \cos \left(\Omega t-q_{m} z+\phi_{2}\right)$, where the modulation amplitude itself also undergoes a spatial modulation

$$
\begin{aligned}
& \Delta n_{1}(z)=\Delta n \cosh \left(F_{2} z\right), \\
& \Delta n_{2}(z)=\Delta n \sinh \left(F_{2} z\right) .
\end{aligned}
$$

where $\Delta n$ is initial modulation amplitude at $z=0$. Additionally $F_{1}=q-q_{m}=-\Delta q$ and $\phi_{2}-\phi_{1}=\pi / 2$ should also hold. So the real part of the force $F_{1}$ is still determined by

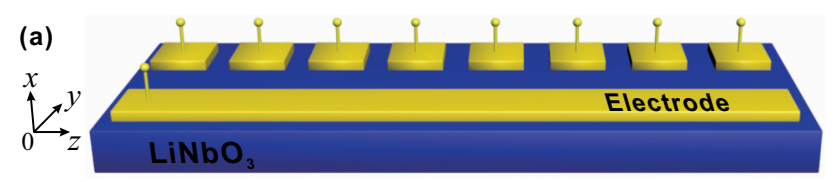

(b) $\uparrow$

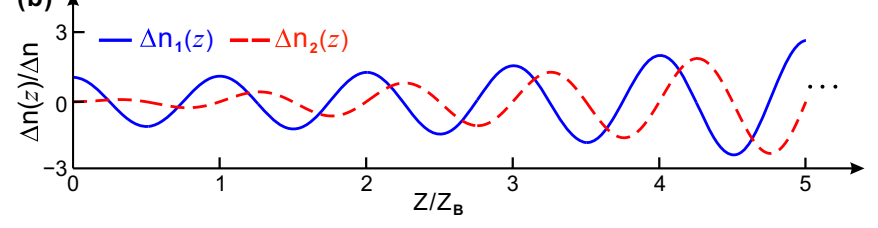

FIG. 7. (a) Schematic modulator structure with periodic electrodes to achieve the complex effective force. (b) Spatial distribution of complex index modulations at initial time $t=0$, where the blue solid and red dashed curves denote the real and imaginary parts, respectively. 
the wave number mismatch, while the imaginary part $F_{2}$ is denoted by the spatial profile of modulation amplitude. As shown in Fig. 7(a), by using periodic electrodes instead of homogeneous electrodes, the modulation amplitudes can be configured point by point so as to fulfill the required modulation profiles. Figure 7(b) depicts the real and imaginary parts of complex index distribution at initial time $t=0$, the shapes of which align with the oscillation trajectory and intensity evolution function shown in Figs. 6(a) and 6(b), respectively.

\section{CONCLUSIONS AND DISCUSSIONS}

In summary, we exploit complex modulation to create nonHermitian frequency lattices and synthesize complex gauge fields to achieve nonconservative frequency diffraction. By using complex gauge potentials, we achieve amplified or decayed discrete diffraction and refraction. In the presence of effective electric force in the lattice, frequency Bloch oscillations can also emerge, where the mode energy also manifests a periodic oscillation or can be exponentially amplified. Finally, we show that Bloch oscillations can provide a bulk probing method to detect the existence (or not) of the NHSE in the lattice without open boundaries. We also provide concrete modulator structures with judiciously designed modulation profiles to achieve the complex photonic gauge fields.

The study provides a paradigm to achieve the effects of non-Hermitian diffraction and Bloch oscillations in synthetic dimension through temporal modulation approaches. This is different from traditional non-Hermitian spatial diffraction and Bloch oscillations in $P T$-symmetric waveguide arrays [12-14] or silicon resonator chains with auxiliary asymmetric coupling elements $[47,48,71,72]$. Compared to spatial nonHermitian lattices requiring precise designs of lattice geometry and gain/loss distribution, our design based on a synthetic lattice realized in an electro-optical controlled $\mathrm{LiNbO}_{3}$ slab waveguide can simplify the system complexity and is dynamically reconfigurable on a fast time scale. Our results also provide functionalities to control both the spectrum and energy evolution of light. For example, the amplified and decayed frequency diffraction and refraction can be applied to the spectrum reshaping, filtering, and asymmetric frequency conversion. This paves the way for applications of nonHermitian frequency Bloch oscillations to spectrum-temporal imaging, optical communications, and signal processing. Finally, Bloch oscillations can probe in the bulk the skin effect arising from asymmetric hopping. The non-Hermitian skin effect is a hallmark of many non-Hermitian topological systems [26-44,73,74], which is attracting a huge attention both from fundamental and applied viewpoints. From a fundamental perspective, the non-Hermitian skin effect characterizes unique topological phases of non-Hermitian systems, where the bulk-boundary correspondence based on Bloch band invariants generally fails to correctly predict topological edge states. From an applied viewpoint, the non-Hermitian skin effect may have potential applications in photonics, for example, for the design of stable topological lasers $[45,46,73]$ and for enhanced optical sensing [74]. The design of a reconfigurable synthetic photonic lattice in frequency domain with asymmetric hopping, suggested in our work, thus provides an interesting route for the realization and probing of topological non-Hermitian phases with tunable skin effect, stimulating further theoretical and experimental investigations in the rapidly growing field of non-Hermitian topological physics.

\section{ACKNOWLEDGMENTS}

The work is supported by the National Natural Science Foundation of China (Grants No. 11674117, No. 11974124, and No. 11947209), National Postdoctoral Program for Innovative Talent (Grant No. BX20190129), and Chinese Postdoctoral Science Foundation General Program (Grant No. 2019M660180). Z.J.W. acknowledges the support from a Texas A\&M University start-up grant.

\section{APPENDIX A: COUPLED-MODE EQUATIONS AND THE SOLVING METHOD}

In this appendix, we present the detailed derivation of coupled-mode Eq. (1) in the main text and provide a matrix algorithm to solve it. Consider the complex index modulation as shown in the main text $n(z, t)=n_{0}+\Delta n_{1} \cos \left(\Omega t-q_{m} z+\phi_{1}\right)+$ $i \Delta n_{2} \cos \left(\Omega t-q_{m} z+\phi_{2}\right)$, the time-varying dielectric permittivity is

$$
\begin{aligned}
\varepsilon(z, t)= & n(z, t)^{2}=\left[n_{0}+\Delta n_{1} \cos \left(\Omega t-q_{m} z+\phi_{1}\right)+i \Delta n_{2} \cos \left(\Omega t-q_{m} z+\phi_{2}\right)\right]^{2} \\
= & n_{0}^{2}+2 n_{0} \Delta n_{1} \cos \left(\Omega t-q_{m} z+\phi_{1}\right)+i 2 n_{0} \Delta n_{2} \cos \left(\Omega t-q_{m} z+\phi_{2}\right) \\
& +\Delta n_{1}^{2} \cos ^{2}\left(\Omega t-q_{m} z+\phi_{1}\right)-\Delta n_{2}^{2} \cos ^{2}\left(\Omega t-q_{m} z+\phi_{2}\right) \\
& +2 i \Delta n_{1} \Delta n_{2} \cos \left(\Omega t-q_{m} z+\phi_{1}\right) \cos \left(\Omega t-q_{m} z+\phi_{2}\right),
\end{aligned}
$$

for weak modulation amplitudes $\Delta n_{1}, \Delta n_{2} \ll n_{0}$, the quadratic terms can be neglected, $\varepsilon(z, t)$ thus reads

$$
\varepsilon(z, t)=\varepsilon_{0}+\Delta \varepsilon_{1} \cos \left(\Omega t-q_{m} z+\phi_{1}\right)+i \Delta \varepsilon_{2} \cos \left(\Omega t-q_{m} z+\phi_{2}\right),
$$

where $\varepsilon_{0}=n_{0}^{2}, \Delta \varepsilon_{1}=2 n_{0} \Delta n_{1}, \Delta \varepsilon_{2}=2 n_{0} \Delta n_{2}$. The electric field is $E(z, t)=\sum_{n} a_{n}(z) \exp \left[i\left(\omega_{n} t-\beta_{n} z\right)\right]$, with $\omega_{n}=\omega_{0}+n \Omega$, $\beta_{n}=\beta_{0}+n q(n=0, \pm 1, \pm 2, \ldots)$, which obeys the time-dependent wave equation

$$
\nabla^{2} E(z, t)-\frac{1}{c^{2}} \frac{\partial^{2}}{\partial t^{2}}[\varepsilon(z, t) E(z, t)]=0
$$


Substituting the electric-field distribution and (A2) into (A3), we can obtain

$$
\frac{\partial^{2}}{\partial z^{2}} E(z, t)-\frac{\varepsilon_{0}}{c^{2}} \frac{\partial^{2}}{\partial t^{2}} E(z, t)=\frac{1}{c^{2}} \frac{\partial^{2}}{\partial t^{2}}\left\{\left[\Delta \varepsilon_{1} \cos \left(\Omega t-q_{m} z+\phi_{1}\right)+i \Delta \varepsilon_{2} \cos \left(\Omega t-q_{m} z+\phi_{2}\right)\right] E(z, t)\right\} .
$$

By applying slowly varying amplitude approximation, the left side of Eq. (A4) is

$$
-\sum_{n} 2 i \beta_{n} \frac{\partial a_{n}(z)}{\partial z} e^{i\left(\omega_{n} t-\beta_{n} z\right)}
$$

Denoting $\omega_{n} \pm \Omega=\omega_{n \pm 1}, \beta_{n} \pm q=\beta_{n \pm 1}$, the right side of Eq. (A4) is

$$
\begin{aligned}
\frac{1}{2 c^{2}} & \frac{\partial^{2}}{\partial t^{2}}\left\{\left[\Delta \varepsilon_{1}\left(e^{i\left(\Omega t-q_{m} z+\phi_{1}\right)}+e^{-i\left(\Omega t-q_{m} z+\phi_{1}\right)}\right)+i \Delta \varepsilon_{2}\left(e^{i\left(\Omega t-q_{m} z+\phi_{2}\right)}+e^{-i\left(\Omega t-q_{m} z+\phi_{2}\right)}\right)\right] \sum_{n} a_{n}(z) e^{i\left(\omega_{n} t-\beta_{n} z\right)}\right\} \\
= & -\frac{1}{2 c^{2}} \sum_{n} a_{n}(z) \Delta \varepsilon_{1}\left[\omega_{n+1}^{2} e^{i\left(\omega_{n+1} t-\beta_{n+1} z\right)} e^{i\left(\Delta q z+\phi_{1}\right)}+\omega_{n-1}^{2} e^{i\left(\omega_{n-1} t-\beta_{n-1} z\right)} e^{-i\left(\Delta q z+\phi_{1}\right)}\right] \\
& -\frac{i}{2 c^{2}} \sum_{n} a_{n}(z) \Delta \varepsilon_{2}\left[\omega_{n+1}^{2} e^{i\left(\omega_{n+1} t-\beta_{n+1} z\right)} e^{i\left(\Delta q z+\phi_{2}\right)}+\omega_{n-1}^{2} e^{i\left(\omega_{n-1} t-\beta_{n-1} z\right)} e^{-i\left(\Delta q z+\phi_{2}\right)}\right],
\end{aligned}
$$

where $\Delta q=q-q_{m}$. By substituting $(n \pm 1)$ by $n$, we have

$$
\sum_{n} a_{n}(z) \omega_{n \pm 1}^{2} e^{i\left(\omega_{n \pm 1} t-\beta_{n \pm 1} z\right)}=\sum_{n} a_{n \mp 1}(z) \omega_{n}^{2} e^{i\left(\omega_{n} t-\beta_{n} z\right)}
$$

By combining Eqs. (A5)-(A7), we can obtain the coupled-mode Eq. (1) in the main text

$$
i \frac{\partial a_{n}(z)}{\partial z}=\left[C_{1} e^{i\left(\Delta q z+\phi_{1}\right)}+i C_{2} e^{i\left(\Delta q z+\phi_{2}\right)}\right] a_{n-1}(z)+\left[C_{1} e^{-i\left(\Delta q z+\phi_{1}\right)}+i C_{2} e^{-i\left(\Delta q z+\phi_{2}\right)}\right] a_{n+1}(z),
$$

where $C_{1}=\Delta \varepsilon_{1} \omega_{n}^{2} /\left(4 \beta_{n} c^{2}\right)=\Delta n_{1} k_{0} / 2, C_{2}=\Delta \varepsilon_{2} \omega_{n}^{2} /\left(4 \beta_{n} c^{2}\right)=\Delta n_{2} k_{0} / 2$. To solve Eq. (A8), we can truncate the mode order to a sufficient large value $n=M$, such that (A8) can be rewritten as a time-dependent Schrödinger equation

$$
i \frac{\partial|\Phi(z)\rangle}{\partial z}=\mathbf{H}(z)|\Phi(z)\rangle
$$

where $|\Phi(z)\rangle=\left[a_{1}(z), a_{2}(z), \ldots, a_{N}(z)\right]^{\mathrm{T}}$ is the eigenstate with $N=2 M+1 . \mathbf{H}(z)$ is the eigenmatrix given by

$\mathbf{H}(z)=\left(\begin{array}{cccccc}0 & C_{1} e^{-i\left(\Delta q z+\phi_{1}\right)}+i C_{2} e^{-i\left(\Delta q z+\phi_{2}\right)} & 0 & \cdots & 0 & 0 \\ C_{1} e^{i\left(\Delta q z+\phi_{1}\right)}+i C_{2} e^{i\left(\Delta q z+\phi_{2}\right)} & 0 & C_{1} e^{-i\left(\Delta q z+\phi_{1}\right)}+i C_{2} e^{-i\left(\Delta q z+\phi_{2}\right)} \ldots & \ldots & 0 \\ 0 & C_{1} e^{i\left(\Delta q z+\phi_{1}\right)}+i C_{2} e^{i\left(\Delta q z+\phi_{2}\right)} & 0 & \cdots & 0 & \vdots \\ \vdots & \vdots & \vdots & \ddots & 0 & C_{1} e^{-i\left(\Delta q z+\phi_{1}\right)}+i C_{2} e^{-i\left(\Delta q z+\phi_{2}\right)} \\ 0 & 0 & 0 & \cdots & 0\end{array}\right)$.

The spectrum evolutions can thus be obtained by numerically solving Eq. (A9).

\section{APPENDIX B: THEORY OF SINGLE-FREQUENCY DIFFRACTION AND REFRACTION}

In this appendix, we give theoretical analysis of the single-frequency diffraction and refraction patterns in Figs. 2(e), 3(c), 3(f), 3(i), and 3(1). For a single-frequency input, it is convenient to analyze the spectrum evolutions from optical phase-modulation perspective, which is different from the band structure analysis for Bloch-wave packet input.

First, we consider the single-frequency diffraction in a single modulator with modulation depth $m_{\varphi}$ and complex gauge potential $\phi+i \kappa$. For the input electric-field distribution $E_{\mathrm{in}}(t)=a_{0} \exp \left(i \omega_{0} t\right)$, the complex modulation imposes a complex phase factor on the field, giving rise to the output field

$$
E_{\text {out }}(t)=a_{0} e^{i \omega_{0} t} e^{i m_{\varphi} \cos (\Omega t+\phi+i \kappa)}=a_{0} e^{i \omega_{0} t} \sum_{n=-\infty}^{\infty} i^{n} J_{n}\left(m_{\varphi}\right) e^{i n(\Omega t+\phi+i \kappa)}=a_{0} \sum_{n=-\infty}^{\infty} J_{n}\left(m_{\varphi}\right) e^{-n \kappa} e^{i n(\phi+\pi / 2)} e^{i\left(\omega_{0}+n \Omega\right) t},
$$

where we have applied Jacobi-Anger expansion. The output amplitude spectrum is thus

$$
\left|a_{n}\right|=a_{0}\left|J_{n}\left(m_{\varphi}\right)\right| e^{-n \kappa} .
$$


So the presence of $\kappa$ imprints an amplified $(n>0$ if $\kappa>0)$ and decayed $(n<0)$ exponent to the traditional symmetric pattern $\left|a_{n}\right|=a_{0}\left|J_{n}\left(m_{\varphi}\right)\right|$, giving rise to an asymmetric pattern in Fig. 2(e).

Then, we analyze the frequency-energy refraction in two cascade modulators with modulation depths $m_{\varphi}, m_{\varphi}^{\prime}$ and complex gauge potentials $\phi+i \kappa, \phi^{\prime}+i \kappa^{\prime}$. For cases (1) and (3) with $\phi=\phi^{\prime}$ (or $\phi \neq \phi^{\prime}$ ) and $\kappa=\kappa^{\prime}$, the output field is uniformly described by

$$
\begin{aligned}
E_{\text {out }}(t) & =a_{0} e^{i \omega_{0} t} e^{i m_{\varphi} \cos (\Omega t+\phi+i \kappa)} e^{i m_{\varphi}^{\prime} \cos \left(\Omega t+\phi^{\prime}+i \kappa\right)}=a_{0} e^{i \omega_{0} t} e^{i \sqrt{m_{\varphi}^{2}+m_{\varphi}^{\prime 2}+2 m_{\varphi} m_{\varphi}^{\prime} \cos \left(\phi^{\prime}-\phi\right)} \cos (\Omega t+\theta+i \kappa)} \\
& =a_{0} e^{i \omega_{0} t} \sum_{n=-\infty}^{\infty} i^{n} J_{n}\left(\sqrt{m_{\varphi}^{2}+m_{\varphi}^{\prime 2}+2 m_{\varphi} m_{\varphi}^{\prime} \cos \left(\phi^{\prime}-\phi\right)}\right) e^{i n(\Omega t+\theta+i \kappa)} \\
& =a_{0} \sum_{n=-\infty}^{\infty} J_{n}\left(\sqrt{m_{\varphi}^{2}+m_{\varphi}^{\prime 2}+2 m_{\varphi} m_{\varphi}^{\prime} \cos \left(\phi^{\prime}-\phi\right)}\right) e^{-n \kappa} e^{i n(\theta+\pi / 2)} e^{i\left(\omega_{0}+n \Omega\right) t},
\end{aligned}
$$

where $\theta=\arctan \left\{\left[m_{\varphi} \sin (\phi)+m_{\varphi}^{\prime} \sin \left(\phi^{\prime}\right)\right] /\left[m_{\varphi} \cos (\phi)+m_{\varphi}^{\prime} \cos \left(\phi^{\prime}\right)\right]\right\}$. The output amplitude spectrum is

$$
\left|a_{n}\right|=a_{0}\left|J_{n}\left(\sqrt{m_{\varphi}^{2}+m_{\varphi}^{\prime 2}+2 m_{\varphi} m_{\varphi}^{\prime} \cos \left(\phi^{\prime}-\phi\right)}\right)\right| e^{-n \kappa} .
$$

Specifically, under the in-phase or out-of-phase modulations, we have

$$
\left|a_{n}\right|=\left\{\begin{array}{ll}
a_{0}\left|J_{n}\left(m_{\varphi}+m_{\varphi}^{\prime}\right)\right| e^{-n \kappa}, & \phi^{\prime}-\phi=0 \\
a_{0}\left|J_{n}\left(m_{\varphi}-m_{\varphi}^{\prime}\right)\right| e^{-n \kappa}, & \phi^{\prime}-\phi=\pi
\end{array} .\right.
$$

Both of which exhibit asymmetric refraction patterns, as shown in the main text Figs. 3(c) and 3(i). Furthermore, if we choose $m_{\varphi}^{\prime}=m_{\varphi}$, we have

$$
\left|a_{n}\right|=\left\{\begin{array}{l}
a_{0}\left|J_{n}\left(2 m_{\varphi}\right)\right| e^{-n \kappa}, \phi^{\prime}-\phi=0 \\
a_{0}\left|\delta_{n, 0}\right| e^{-n \kappa}, \phi^{\prime}-\phi=\pi
\end{array} .\right.
$$

So the out-of-phase modulation can realize the spectrum perfect imaging, as shown in Fig. 3(i).

Next, we consider the case (2) with $\phi=\phi^{\prime}$ and $\kappa \neq \kappa^{\prime}$, the output field distribution is

$$
\begin{aligned}
E_{\text {out }}(t) & =a_{0} e^{i \omega_{0} t} e^{i m_{\varphi} \cos (\Omega t+\phi+i \kappa)} e^{i m_{\varphi}^{\prime} \cos \left(\Omega t+\phi+i \kappa^{\prime}\right)}=a_{0} e^{i \omega_{0} t} e^{i\left[m_{\varphi} \cosh (\kappa)+m_{\varphi}^{\prime} \cosh \left(\kappa^{\prime}\right)\right] \cos (\Omega t+\phi)} e^{\left[m_{\varphi} \sinh (\kappa)+m_{\varphi}^{\prime} \sinh \left(\kappa^{\prime}\right)\right] \sin (\Omega t+\phi)} \\
& =a_{0} e^{i \omega_{0} t} \sum_{n=-\infty}^{\infty} J_{n}\left[m_{\varphi} \cosh (\kappa)+m_{\varphi}^{\prime} \cosh \left(\kappa^{\prime}\right)\right] e^{i n(\Omega t+\phi+\pi / 2)} \sum_{n=-\infty}^{\infty} I_{n}\left[-m_{\varphi} \sinh (\kappa)-m_{\varphi}^{\prime} \sinh \left(\kappa^{\prime}\right)\right] e^{i n(\Omega t+\phi+\pi / 2)} \\
& =a_{0} \sum_{n=-\infty}^{\infty} \sum_{m=-\infty}^{\infty} J_{m}\left[m_{\varphi} \cosh (\kappa)+m^{\prime}{ }_{\varphi} \cosh \left(\kappa^{\prime}\right)\right] I_{n-m}\left[-m_{\varphi} \sinh (\kappa)-m_{\varphi}^{\prime} \sinh \left(\kappa^{\prime}\right)\right] e^{i n(\phi+\pi / 2)} e^{i\left(\omega_{0}+n \Omega\right) t},
\end{aligned}
$$

where $I_{n}$ is $n$ th-order modified Bessel function. In above derivation, we have exploited the Cauchy product. The output amplitude spectrum thus reads

$$
\left|a_{n}\right|=a_{0}\left|\sum_{m=-\infty}^{\infty} J_{m}\left[m_{\varphi} \cosh (\kappa)+m_{\varphi}^{\prime} \cosh \left(\kappa^{\prime}\right)\right] I_{n-m}\left[-m_{\varphi} \sinh (\kappa)-m_{\varphi}^{\prime} \sinh \left(\kappa^{\prime}\right)\right]\right| .
$$

Specifically, if we choose $\kappa^{\prime}=-\kappa$ and $m_{\varphi}=m_{\varphi}^{\prime}$, as shown in the main text Fig. 3(f), the output spectrum reduces to a symmetric function

$$
\left|a_{n}\right|=a_{0}\left|\sum_{m=-\infty}^{\infty} J_{m}\left[2 m_{\varphi} \cosh (\kappa)\right] I_{n-m}(0)\right|=a_{0}\left|J_{n}\left[2 m_{\varphi} \cosh (\kappa)\right]\right| .
$$

Finally, we consider case (4) with $\phi \neq \phi^{\prime}$ and $\kappa \neq \kappa^{\prime}$, the output field is

$$
\begin{aligned}
E_{\mathrm{out}}(t) & =a_{0} e^{i \omega_{0} t} e^{i m_{\varphi} \cos (\Omega t+\phi+i \kappa)} e^{i m_{\varphi}^{\prime} \cos \left(\Omega t+\phi^{\prime}+i \kappa^{\prime}\right)}=a_{0} e^{i \omega_{0} t} e^{i[a \cos (\Omega t)-b \sin (\Omega t)]} e^{i[c \sin (\Omega t)+d \cos (\Omega t)]} \\
& =a_{0} e^{i \omega_{0} t} e^{i \sqrt{a^{2}+b^{2}} \cos \left(\Omega t+\theta_{1}\right)} e^{\sqrt{c^{2}+d^{2}} \sin \left(\Omega t+\theta_{2}\right)} \\
& =a_{0} e^{i \omega_{0} t} \sum_{n=-\infty}^{\infty} J_{n}\left(\sqrt{a^{2}+b^{2}}\right) e^{i n\left(\Omega t+\theta_{1}+\pi / 2\right)} \sum_{n=-\infty}^{\infty} I_{n}\left(-\sqrt{c^{2}+d^{2}}\right) e^{i n\left(\Omega t+\theta_{2}+\pi / 2\right)} \\
& =a_{0} \sum_{n=-\infty}^{\infty}\left(\sum_{m=-\infty}^{\infty} J_{m}\left(\sqrt{a^{2}+b^{2}}\right) I_{n-m}\left(-\sqrt{c^{2}+d^{2}}\right) e^{i m\left(\theta_{2}-\theta_{2}\right)}\right) e^{i n\left(\theta_{2}+\pi / 2\right)} e^{i\left(\omega_{0}+n \Omega\right) t},
\end{aligned}
$$


where $\theta_{1}=\arctan (b / a), \theta_{2}=\arctan (d / c)$, with

$$
\begin{aligned}
a & =m_{\varphi} \cosh (\kappa) \cos (\phi)+m_{\varphi}^{\prime} \cosh \left(\kappa^{\prime}\right) \cos \left(\phi^{\prime}\right), \\
b & =m_{\varphi} \cosh (\kappa) \sin (\phi)+m_{\varphi}^{\prime} \cosh \left(\kappa^{\prime}\right) \sin \left(\phi^{\prime}\right), \\
c & =m_{\varphi} \sinh (\kappa) \cos (\phi)+m_{\varphi}^{\prime} \sinh \left(\kappa^{\prime}\right) \cos \left(\phi^{\prime}\right), \\
d & =m_{\varphi} \sinh (\kappa) \sin (\phi)+m_{\varphi}^{\prime} \sinh \left(\kappa^{\prime}\right) \sin \left(\phi^{\prime}\right) .
\end{aligned}
$$

So the output amplitude spectrum reads

$$
\left|a_{n}\right|=a_{0}\left|\sum_{m=-\infty}^{\infty} J_{m}\left(\sqrt{a^{2}+b^{2}}\right) I_{n-m}\left(-\sqrt{c^{2}+d^{2}}\right) e^{i m\left(\theta_{2}-\theta_{2}\right)}\right| .
$$

The spectrum pattern is shown in Fig. 3(1).

\section{APPENDIX C: DERIVATION OF AMPLIFIED BLOCH OSCILLATIONS}

In this appendix, we derive the frequency shift and intensity evolution for the amplified non-Hermitian Bloch oscillations. From Eq. (16) in the main text, the frequency shift is

$$
\begin{aligned}
\Delta \omega(z)= & \int_{0}^{z} v_{g, \omega}\left(z^{\prime}\right) d z^{\prime}=\int_{0}^{z}-2 C \Omega \cosh \left(F_{2} z^{\prime}\right) \sin \left(\phi_{0}-\phi-F_{1} z^{\prime}\right) d z^{\prime} \\
= & C \Omega\left\{\cos \left(\phi-\phi_{0}\right) \int_{0}^{z}\left[e^{F_{2} z^{\prime}} \sin \left(F_{1} z^{\prime}\right)+e^{-F_{2} z^{\prime}} \sin \left(F_{1} z^{\prime}\right)\right] d z^{\prime}+\sin \left(\phi-\phi_{0}\right) \int_{0}^{z}\left[e^{F_{2} z^{\prime}} \cos \left(F_{1} z^{\prime}\right)+e^{-F_{2} z^{\prime}} \cos \left(F_{1} z^{\prime}\right)\right] d z^{\prime}\right\} \\
= & C \Omega \cos \left(\phi-\phi_{0}\right)\left\{\left.\frac{e^{F_{2} z^{\prime}}\left[F_{2} \sin \left(F_{1} z^{\prime}\right)-F_{1} \cos \left(F_{1} z^{\prime}\right)\right]}{F_{1}^{2}+F_{2}^{2}}\right|_{0} ^{z}+\left.\frac{e^{-F_{2} z^{\prime}}\left[-F_{2} \sin \left(F_{1} z^{\prime}\right)-F_{1} \cos \left(F_{1} z^{\prime}\right)\right]}{F_{1}^{2}+F_{2}^{2}}\right|_{0} ^{z}\right\} \\
& +C \Omega \sin \left(\phi-\phi_{0}\right)\left\{\left.\frac{e^{F_{2} z^{\prime}}\left[F_{2} \cos \left(F_{1} z^{\prime}\right)+F_{1} \sin \left(F_{1} z^{\prime}\right)\right]}{F_{1}^{2}+F_{2}^{2}}\right|_{0} ^{z}+\left.\frac{e^{-F_{2} z^{\prime}}\left[-F_{2} \cos \left(F_{1} z^{\prime}\right)+F_{1} \sin \left(F_{1} z^{\prime}\right)\right]}{F_{1}^{2}+F_{2}^{2}}\right|_{0} ^{z}\right\} \\
= & \frac{2 C \Omega}{F_{1}^{2}+F_{2}^{2}}\left[F_{2} \sinh \left(F_{2} z\right) \sin \left(\phi_{1}-\phi_{0}+F_{1} z\right)-F_{1} \cosh \left(F_{2} z\right) \cos \left(\phi_{1}-\phi_{0}+F_{1} z\right)+F_{1} \cos \left(\phi_{1}-\phi_{0}\right)\right],
\end{aligned}
$$

in which we have applied the identities of integrals

$$
\begin{aligned}
& \int e^{a x} \cos (b x) d x=\frac{e^{a x}[a \cos (b x)+b \sin (b x)]}{a^{2}+b^{2}}, \\
& \int e^{a x} \sin (b x) d x=\frac{e^{a x}[a \sin (b x)-b \cos (b x)]}{a^{2}+b^{2}} .
\end{aligned}
$$

Similarly, we can also derivate the intensity evolution

$$
I(z)=I(0) \exp \left(4 C \int_{0}^{z} \sinh \left(F_{2} z^{\prime}\right) \sin \left(k_{\omega} \Omega-\phi-\Delta q z^{\prime}\right) d z^{\prime}\right),
$$

where the integration is given by

$$
\begin{aligned}
& \int_{0}^{z} \sinh \left(F_{2} z^{\prime}\right) \sin \left(\phi_{0}-\phi-F_{1} z^{\prime}\right) d z^{\prime} \\
& =\frac{1}{2}\left\{\sin \left(\phi_{0}-\phi\right) \int_{0}^{z}\left[e^{F_{2} z^{\prime}} \cos \left(F_{1} z^{\prime}\right)-e^{-F_{2} z^{\prime}} \cos \left(F_{1} z^{\prime}\right)\right] d z^{\prime}-\cos \left(\phi_{0}-\phi\right) \int_{0}^{z}\left[e^{F_{2} z^{\prime}} \sin \left(F_{1} z^{\prime}\right)-e^{-F_{2} z^{\prime}} \sin \left(F_{1} z^{\prime}\right)\right] d z^{\prime}\right\} \\
& =\frac{1}{2} \sin \left(\phi_{0}-\phi\right)\left\{\left.\frac{e^{F_{2} z^{\prime}}\left[F_{2} \cos \left(F_{1} z^{\prime}\right)+F_{1} \sin \left(F_{1} z^{\prime}\right)\right]}{F_{1}^{2}+F_{2}^{2}}\right|_{0} ^{z}-\left.\frac{e^{-F_{2} z^{\prime}}\left[-F_{2} \cos \left(F_{1} z^{\prime}\right)+F_{1} \sin \left(F_{1} z^{\prime}\right)\right]}{F_{1}^{2}+F_{2}^{2}}\right|_{0} ^{z}\right\} \\
& -\frac{1}{2} \cos \left(\phi_{0}-\phi\right)\left\{\left.\frac{e^{F_{2} z^{\prime}}\left[F_{2} \sin \left(F_{1} z^{\prime}\right)-F_{1} \cos \left(F_{1} z^{\prime}\right)\right]}{F_{1}^{2}+F_{2}^{2}}\right|_{0} ^{z}-\left.\frac{e^{-F_{2} z^{\prime}}\left[-F_{2} \sin \left(F_{1} z^{\prime}\right)-F_{1} \cos \left(F_{1} z^{\prime}\right)\right]}{F_{1}^{2}+F_{2}^{2}}\right|_{0} ^{z}\right\} \\
& =\frac{1}{F_{1}^{2}+F_{2}^{2}}\left[F_{2} \cosh \left(F_{2} z\right) \sin \left(\phi_{0}-\phi-F_{1} z\right)+F_{1} \sinh \left(F_{2} z\right) \cos \left(\phi_{0}-\phi-F_{1} z\right)-F_{2} \sin \left(\phi_{0}-\phi\right)\right] \text {. }
\end{aligned}
$$


So the intensity is given by $I(z)=I_{0} \exp [\alpha(z)]$, with $\alpha(z)$ given by

$$
\alpha(z)=\frac{4 C}{F_{1}^{2}+F_{2}^{2}}\left[F_{2} \cosh \left(F_{2} z\right) \sin \left(\phi_{0}-\phi-F_{1} z\right)+F_{1} \sinh \left(F_{2} z\right) \cos \left(\phi_{0}-\phi-F_{1} z\right)-F_{2} \sin \left(\phi_{0}-\phi\right)\right] .
$$

The results of Eqs. (C4) and (C5) are given by Eqs. (17) and (18) in the main text.

[1] C. Minot, J. M. Moison, S. Guilet, E. Cambril, A. Levenson, and N. Belabas, Opt. Lett. 42, 539 (2017).

[2] A. Poddubny, I. Iorsh, P. Belov, and Y. Kivshar, Nat. Photonics 7, 948 (2013).

[3] D. N. Christodoulides, F. Lederer, and Y. Silberberg, Nature (London) 424, 817 (2003).

[4] I. L. Garanovich, S. Longhi, A. A. Sukhorukov, and Y. S. Kivshar, Phys. Rep. 518, 1 (2012).

[5] M. C. Rechtsman, J. M. Zeuner, Y. Plotnik, Y. Lumer, D. Podolsky, F. Dreisow, S. Nolte, M. Segev, and A. Szameit, Nature 496, 196 (2013).

[6] H. S. Eisenberg, Y. Silberberg, R. Morandotti, and J. S. Aitchison, Phys. Rev. Lett. 85, 1863 (2000).

[7] T. Pertsch, T. Zentgraf, U. Peschel, A. Bräuer, and F. Lederer, Phys. Rev. Lett. 88, 093901 (2002).

[8] B. Wang, X. Zhang, F. J. García-Vidal, X. Yuan, and J. Teng, Phys. Rev. Lett. 109, 073901 (2012).

[9] T. Pertsch, P. Dannberg, W. Elflein, A. Bräuer, and F. Lederer, Phys. Rev. Lett. 83, 4752 (1999).

[10] A. Joushaghani, R. Iyer, J. K. S. Poon, J. S. Aitchison, C. M. de Sterke, J. Wan, and M. M. Dignam, Phys. Rev. Lett. 103, 143903 (2009).

[11] A. Block, C. Etrich, T. Limboeck, F. Bleckmann, E. Soergel, C. Rockstuhl, and S. Linden, Nat. Commun. 5, 3843 (2014).

[12] T. Eichelkraut, R. Heilmann, S. Weimann, S. Stutzer, F. Dreisow, D. N. Christodoulides, S. Nolte, and A. Szameit, Nat. Commun. 4, 2533 (2013).

[13] S. Longhi, Phys. Rev. Lett. 103, 123601 (2009).

[14] Y. Xu, W. Fegadolli, L. Gan, M. Lu, X. Liu, Z. Li, A. Scherer, and Y. Chen, Nat. Commun. 7, 11319 (2016).

[15] J. W. Yoon, G. M. Koh, S. H. Song, and R. Magnusson, Phys. Rev. Lett. 109, 257402 (2012).

[16] Z. J. Wong, Y.-L. Xu, J. Kim, K. O’Brien, Y. Wang, L. Feng, and X. Zhang, Nat. Photonics 10, 796 (2016).

[17] M. P. Hokmabadi, N. S. Nye, R. El-Ganainy, D. N. Christodoulides, and M. Khajavikhan, Science 363, 623 (2019).

[18] H. Xu, D. Mason, L. Jiang, and J. G. Harris, Nature (London) 537, 80 (2016).

[19] J. Doppler, A. A. Mailybaev, J. Böhm, U. Kuhl, A. Girschik, F. Libisch, T. J. Milburn, P. Rabl, N. Moiseyev, and S. Rotter, Nature (London) 537, 76 (2016).

[20] S. Assawaworrarit, X. Yu, and S. Fan, Nature (London) 546, 387 (2017).

[21] K. G. Makris, R. El-Ganainy, D. N. Christodoulides, and Z. H. Musslimani, Phys. Rev. Lett. 100, 103904 (2008).

[22] C. E. Rüter, K. G. Makris, R. El-Ganainy, D. N. Christodoulides, M. Segev, and D. Kip, Nat. Phys. 6, 192 (2010).
[23] A. Regensburger, C. Bersch, M.-A. Miri, G. Onishchukov, D. N. Christodoulides, and U. Peschel, Nature (London) 488, 167 (2012).

[24] N. Hatano and D. R. Nelson, Phys. Rev. Lett. 77, 570 (1996).

[25] N. Hatano and D. R. Nelson, Phys. Rev. B 58, 8384 (1998).

[26] Z. Gong, Y. Ashida, K. Kawabata, K. Takasan, S. Higashikawa, and M. Ueda, Phys. Rev. X 8, 031079 (2018).

[27] Y. Xiong, J. Phys. Commun. 2, 035043 (2018).

[28] F. K. Kunst, E. Edvardsson, J. C. Budich, and E. J. Bergholtz, Phys. Rev. Lett. 121, 026808 (2018).

[29] S. Yao and Z. Wang, Phys. Rev. Lett. 121, 086803 (2018).

[30] S. Yao, F. Song, and Z. Wang, Phys. Rev. Lett. 121, 136802 (2018).

[31] C. H. Lee and R. Thomale, Phys. Rev. B 99, 201103(R) (2019).

[32] K. Yokomizo and S. Murakami, Phys. Rev. Lett. 123, 066404 (2019).

[33] A. Ghatak and T. Das, J. Phys.: Condens. Matter 31, 263001 (2019).

[34] S. Longhi, Phys. Rev. Res. 1, 023013 (2019).

[35] L. Jin and Z. Song, Phys. Rev. B 99, 081103(R) (2019).

[36] D. S. Borgnia, A. J. Kruchkov, and R.-J. Slager, arXiv:1902.07217v2 (2019).

[37] F. Song, S. Yao, and Z. Wang, Phys. Rev. Lett. 123, 170401 (2019).

[38] L. Xiao, T. Deng, K.Wang, G. Zhu, Z. Wang, W. Yi, and P. Xue, arXiv:1907.12566 (2019).

[39] T. Hofmann, T. Helbig, F. Schindler, N. Salgo, M. Brzezińska, M. Greiter, T. Kiessling, D. Wolf, A. Vollhardt, A. Kabaši, C. H. Lee, A. Bilušić, R. Thomale, and T. Neupert, arXiv:1908.02759v1 (2019).

[40] T. Helbig, T. Hofmann, S. Imhof, M. Abdelghany, T. Kiessling, L. W. Molenkamp, C. H. Lee, A. Szameit, M. Greiter, and R. Thomale, arXiv:1907.11562 (2019).

[41] A. Ghatak, M. Brandenbourger, J. van Wezel, and C. Coulais, arXiv:1907.11619 (2019).

[42] K. Zhang, Z. Yang, and C. Fang, arXiv:1910.01131 (2019).

[43] N. Okuma, K. Kawabata, K. Shiozaki, and M. Sato, arXiv:1910.02878 (2019).

[44] E. J. Bergholtz, J. C. Budich, and F. K. Kunst, arXiv:1912.10048 (2019).

[45] S. Longhi, Ann. Phys. (Berlin) 530, 1800023 (2018).

[46] S. Longhi and L. Feng, APL Photonics 3, 060802 (2018).

[47] S. Longhi, D. Gatti, and G. Della Valle, Sci Rep 5, 13376 (2015).

[48] S. Longhi, D. Gatti, and G. Della Valle, Phys. Rev. B 92, 094204 (2015).

[49] S. Longhi, Opt. Lett. 30, 786 (2005). 
[50] C. Bersch, G. Onishchukov, and U. Peschel, Appl. Phys. B 104, 495 (2011).

[51] B. A. Bell, K. Wang, A. S. Solntsev, D. N. Neshev, A. A. Sukhorukov, and B. J. Eggleton, Optica 4, 1433 (2017).

[52] C. Qin, F. Zhou, Y. Peng, D. Sounas, X. Zhu, B. Wang, J. Dong, X. Zhang, A. Alù, and P. Lu, Phys. Rev. Lett. 120, 133901 (2018).

[53] C. Qin, L. Yuan, B. Wang, S. Fan, and P. Lu, Phys. Rev. A 97, 063838 (2018).

[54] L. Yuan, Q. Lin, M. Xiao, and S. Fan, Optica 5, 1396 (2018).

[55] U. Peschel, C. Bersch, and G. Onishchukov, Cent. Eur. J. Phys. 6, 3 (2008).

[56] H. Chen, C. Qin, B. Wang, and P. Lu, Opt. Lett. 44, 363 (2019).

[57] X. W. Luo, X. Zhou, J. S. Xu, C. F. Li, G. C. Guo, C. Zhang, and Z. W. Zhou, Nat. Commun. 8, 16097 (2017).

[58] L. Yuan, Q. Lin, A. Zhang, M. Xiao, X. Chen, and S. Fan, Phys. Rev. Lett. 122, 083903 (2019).

[59] S. Longhi, Phys. Rev. A 92, 042116 (2015).

[60] S. Longhi, Phys. Rev. E 64, 047201 (2001).

[61] S. Longhi, Europhys. Lett. 106, 34001 (2014).

[62] S. Longhi, Opt. Lett. 44, 1190 (2019).
[63] J. B. Pendry, Phys. Rev. Lett. 85, 3966 (2000).

[64] R. Salem, M. A. Foster, and A. L. Gaeta, Adv. Opt. Photon. 5, 274 (2013).

[65] J. M. Lukens, D. E. Leaird, and A. W. Weiner, Nature (London) 498, 205 (2013).

[66] C. Qin, B. Wang, and P. Lu, Opt. Express 26, 25721 (2018).

[67] C. Qin, Q. Liu, B. Wang, and P. Lu, Opt. Express 26, 20929 (2018).

[68] W. Li, C. Qin, T. Han, H. Chen, B. Wang, and P. Lu, Opt. Lett. 44, 5430 (2019).

[69] Z. Yang, W. Cao, X. Chen, J. Zhang, Y. Mo, H. Xu, K. Mi, Q. Zhang, P. Lan, and P. Lu, Opt. Lett. 45, 567 (2020).

[70] B. Wang, T. Han, H. Chen, W. Li, and P. Lu, J. Lightwave Technol., 1 (2020).

[71] M. Hafezi, E. A. Demler, M. D. Lukin, and J. M. Taylor, Nat. Phys. 7, 907 (2011).

[72] M. Hafezi, S. Mittal, J. Fan, A. Migdall, and J. M. Taylor, Nat. Photonics. 7, 1001 (2013).

[73] F. Song, S. Yao, and Z. Wang, Phys. Rev. Lett. 123, 246801 (2019).

[74] S. Longhi, Ann. Phys. (Berlin) 531, 1900054 (2019). 